\title{
A Simple and Practical Theoretical Model for Interpreting Binary Azeotropes
}

Jingsong Huang,,${ }^{\text {a, }}$ Panchapakesan Ganesh, ${ }^{a}$ Bobby G. Sumpter, ${ }^{\text {a }}$ David S. Sholl, ,c Kunlun Hong $^{\text {a }}$

${ }^{a}$ Center for Nanophase Materials Sciences, Oak Ridge National Laboratory, Oak Ridge, TN 37831, USA

${ }^{\mathrm{b}}$ School of Chemical \& Biomolecular Engineering, Georgia Institute of Technology, Atlanta, GA 30318, USA

${ }^{c}$ Transformational Decarbonization Initiative, Oak Ridge National Laboratory, Oak Ridge, TN 37831, USA

E-mail: huangj3@ornl.gov

This manuscript has been authored by UT-Battelle, LLC, under contract DE-AC05-00OR22725 with the US Department of Energy (DOE). The US government retains and the publisher, by accepting the article for publication, acknowledges that the US government retains a nonexclusive, paid-up, irrevocable, worldwide license to publish or reproduce the published form of this manuscript, or allow others to do so, for US government purposes. DOE will provide public access to these results of federally sponsored research in accordance with the DOE Public Access Plan (http://energy.gov/downloads/doe-public-access-plan). 
Abstract: We put forth a simple and yet practical theoretical model generalized from Raoult's law and Henry's law and show that it can be reduced to these two laws under limiting conditions. The model entertains a hybrid parameter $h_{B}$ with activity coefficient bundled into it, which smoothly bridges the $p_{B}^{*}$ and $K_{B}$ coefficients in Raoult's law and Henry's law. The value of $h_{B}$ falls in the interval of $\left[K_{B}, p_{B}^{*}\right]$ or $\left[p_{B}^{*}, K_{B}\right]$ in the case of negative or positive deviation from Raoult's law, respectively. We uncover an overlapping rule for the ranges of $h_{A}$ and $h_{B}$, which binary mixtures must obey to form azeotropes. We also provide straightforward ways to analyze the characteristic mole fraction and pressure for azeotropes and to understand the relative positions of vapor composition curves with respect to the liquid counterparts. We rely heavily on experimental data available in the literature for representative binary mixtures with both negative and positive deviations from Raoult's law to illustrate the algebraic derivations. The knowledge gained is useful in the analysis of experimental data from vapor-liquid equilibrium measurements and possess pedagogical merit in various relevant fields.

Keywords: Azeotrope, Raoult's law, Henry's law, vapor-liquid equilibrium, theoretical modeling

\section{Introduction}

Azeotropes are liquid mixtures of two or more components with constant boiling points under a certain pressure at which the compositions of the vapor and liquid phases are identical to each other. ${ }^{1}$ There are two types of azeotropes including maximum-boiling and minimum-boiling azeotropes. $^{2}$ If the experimental control variable is changed from temperature to pressure, these two kinds of azeotropes change to minimum-pressure and maximum-pressure azeotropes, which are also called negative and positive azeotropes, due to their negative and positive deviations from

ideal solutions, respectively. ${ }^{3}$ Azeotropes can impose limits on the separation and purification of 
liquid mixtures by simple distillation. A well-known example is the binary ethanol-water mixture, which is impossible to fully separate by distillation because the condensate under atmospheric pressure is an azeotrope containing $95.6 \mathrm{wt} . \%$ ethanol and $4.4 \mathrm{wt} . \%$ water that prevents further separation by distillation. ${ }^{4}$ Absolute ethanol with $99-100$ wt.\% purity is usually made by using an entrainer such as benzene to break the azeotrope. Despite this downside, azeotropes are used for a wide range of practical applications, such as fragrance, mosquito repellent, cleaning fluids, waste minimization, and azeotropic freeze-drying. ${ }^{5,6,7,8}$ However, even with the long history of azeotropes, their formation mechanism is still not fully understood. ${ }^{9}$

Scientists have carried out various experimental and theoretical studies to gain a fundamental understanding of azeotropes. Experimental studies focused on acquiring and analyzing vapor-liquid equilibrium (VLE) data ${ }^{10,11,12,13}$ and investigating the microstructures of azeotropes and their link to thermodynamics properties. ${ }^{9}, 14$ Theoretical studies ranged from mathematical modeling and statistical mechanics ${ }^{9,15}$ to theoretical predictions of azeotropes using Monte Carlo simulations. ${ }^{16,17}$ All these efforts provided critical insight into the formation mechanism of azeotropes in terms of intermolecular interactions. ${ }^{9}$ However, some questions remain unanswered even for the simplest phase diagrams. For instance, Fig. 1 shows isothermal vapor-liquid phase diagrams for four representative binary mixtures. It is qualitatively understood that tetrachloromethane-tetrahydrofuran and isopropanol-acetone do not form azeotropes due to their small negative and positive deviations from ideality, whereas acetone-chloroform and acetone-carbon disulfide form minimum-pressure and maximum-pressure azeotropes due to their large negative and positive deviations from ideality. However, it is still unclear how the deviations are quantified to be small and large and whether there is a simple guideline to interpret why some solutions form azeotropes while others do not. 

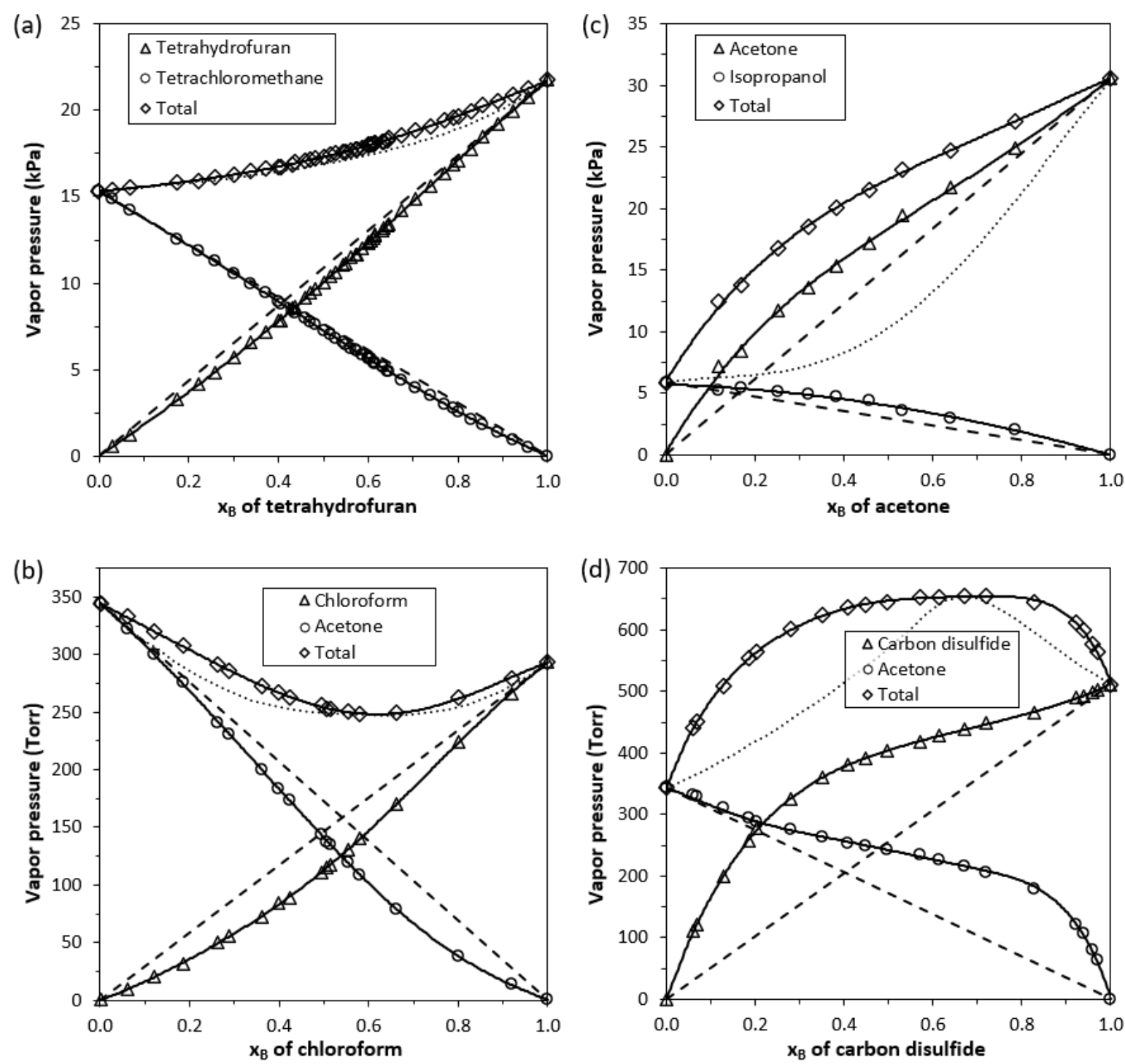

Figure 1. Isothermal partial and total vapor pressures for representative binary mixtures. (a) Tetrachloromethane-tetrahydrofuran $\left\{x_{A} \mathrm{CCl}_{4}+x_{B} \mathrm{C}_{4} \mathrm{H}_{8} \mathrm{O}\right\}$ at $298.15 \mathrm{~K}$. (b) Acetone-chloroform $\left\{x_{A} \mathrm{C}_{3} \mathrm{H}_{6} \mathrm{O}+x_{B} \mathrm{CHCl}_{3}\right\}$ at $308.32 \mathrm{~K}$. (c) Isopropanol-acetone $\left\{x_{A} \mathrm{C}_{3} \mathrm{H}_{8} \mathrm{O}+x_{B} \mathrm{C}_{3} \mathrm{H}_{6} \mathrm{O}\right\}$ at 298.15 K. (d) Acetone-carbon disulfide $\left\{x_{A} \mathrm{C}_{3} \mathrm{H}_{6} \mathrm{O}+x_{B} \mathrm{CS}_{2}\right\}$ at $308.32 \mathrm{~K}$. Data for (a) and (c) are taken from refs. ${ }^{10,11}$ and data for (b) and (d) are taken from ref. ${ }^{12}$. Dashed lines represent Raoult's law and dotted lines indicate vapor compositions under the same total pressures. (a) and (b) show negative deviations while (c) and (d) show positive deviations from Raoult's law. (a) and (c) do not form azeotropes while (b) and (d) form negative and positive azeotropes, respectively. 
Motivated by these open questions, we present a simple and yet practical mathematical interpretation to the isothermal phase diagrams of binary mixtures, which can be easily understood and readily used for data analysis. To that end, we carried out algebraic derivations to 1) put forth a theoretical model entertaining a hybrid parameter $h_{B}$ generalized from Raoult's law and Henry's law and prove that it can be reduced to these two laws under limiting conditions, 2) uncover an overlapping rule for the ranges of $h_{A}$ and $h_{B}$ out of three possible overlapping patterns for binary solutions to form azeotropes, 3) illustrate a simple way to rationalize the characteristic composition and pressure for azeotropes based on their partial and total pressures, and 4) interpret the relative mole fractions of vapor composition curves with respect to the liquid counterparts under the same pressures. Throughout the discussions the hybrid parameter $h_{B}$ plays a central role. To the best of our knowledge, the main conclusions from this work are new and can be adopted as a framework to calibrate experimental results and explain fundamental concepts, e.g., in the textbook Atkins' Physical Chemistry, ${ }^{18}$ in the open access textbook in the chemistry library of the LibreTexts project., ${ }^{19}$ and in the LearnChemE chemical engineering education resources. ${ }^{20}$

\section{Methodologies}

We confined our discussions to the isothermal phase diagrams of generic binary A-B mixtures that form negative and positive azeotropes with a single minimum-pressure or maximum-pressure point. Isobaric phase diagrams using temperature as the control parameter, more complicated multinary azeotropes containing more than two components, and less-common double azeotropes that display two extrema including both a minimum and a maximum are out of the scope of this work. We carried out simple algebraic derivations and relied on available experimental data to illustrate new understanding for VLE measurements. Experimental data were digitized from 
figures or directly taken from tables in the literature. Digitization may introduce some small relative errors, which are typically less than $1 \%$ and therefore too small to affect the conclusions drawn from this work. In cases where necessary, experimental data were fitted by polynomials of order 6 and then the analytical formulae for the first-order derivatives were obtained. An excel file is provided in the Supplementary Materials to show all the detailed steps for data analysis using the experimental data of binary dimethoxymethane-carbon disulfide solution $\left\{x_{A} \mathrm{C}_{3} \mathrm{H}_{8} \mathrm{O}_{2}+x_{B} \mathrm{CS}_{2}\right\}$ at $308.32 \mathrm{~K}$ taken from refs. ${ }^{12,21}$. Additional statistical mechanics explanations of activity, activity coefficient, and the hybrid parameter in terms of intermolecular interactions were also given in the Supplementary Materials.

\section{Results and Discussion}

3.1. Generalization of Raoult's law and Henry's law. Both Raoult's law and Henry's law deal with VLE. Raoult's law states that the partial pressure of each component in a vapor mixture at equilibrium with a liquid mixture is equal to the vapor pressure of the pure component multiplied by its mole fraction in the liquid mixture. Henry's law states that the amount of gas dissolved in a liquid is proportional to its partial pressure above the liquid, by a factor now known as the Henry's law constant. In principle, both laws are applicable to various multinary systems, but herein we will confine our discussions to generic binary A-B mixtures. For ideal solutions, Raoult's law and Henry's law become identical to each other where the Henry's law constant is equal to the equilibrium vapor pressure for the pure component, and they are applicable in the entire range of solution compositions. However, most real solutions are non-ideal and exhibit negative or positive deviations from Raoult's law; hence leading to a Henry's law constant that is different from the equilibrium vapor pressure for the pure component. Under such a circumstance, Raoult's law and 
Henry's law are appliable to only dilute solutions. More specifically, Raoult's law is applicable to only the solvent while Henry's law is applicable only to the solute.

For a binary dilute solution consisting of solvent A and solute B, Raoult's law and Henry's law can be written as:

$$
\begin{aligned}
& p_{A}=p_{A}^{*} x_{A}^{(l)} \\
& p_{B}=K_{B} x_{B}^{(l)}
\end{aligned}
$$

where $p_{A}$ and $p_{B}$ are the partial pressures in the vapor for solvent $\mathrm{A}$ and solute $\mathrm{B}, p_{A}^{*}$ and $K_{B}$ are the vapor pressure of pure solvent A and the Henry's law constant for solute $\mathrm{B}$, and $x_{A}^{(l)}$ and $x_{B}^{(l)}$ are the mole fractions of solvent A and solute B in the liquid phase, respectively. The conditions that $\mathrm{A}$ is solvent and B is solute imply that $x_{A}^{(l)} \rightarrow 1$ and $x_{B}^{(l)} \rightarrow 0$. Conversely, if B is solvent and A is solute, i.e., $x_{B}^{(l)} \rightarrow 1$ and $x_{A}^{(l)} \rightarrow 0$, eqns 1 and 2 only change their format by swapping the subscripts A and B:

$$
\begin{aligned}
& p_{B}=p_{B}^{*} x_{B}^{(l)} \\
& p_{A}=K_{A} x_{A}^{(l)}
\end{aligned}
$$

Since only two components exist in the liquid phase for a binary mixture, the sum of $x_{A}^{(l)}$ and $x_{B}^{(l)}$ is unity, regardless of whether A and B are solvent or solute:

$$
x_{A}^{(l)}+x_{B}^{(l)}=1
$$

and the total pressure in the vapor phase is the sum of the partial pressures based on Dalton's law:

$$
p_{\text {tot }}=p_{A}+p_{B}
$$

Eqns 5 and 6 hold true in the entire range of mole fractions. For convenience of subsequent discussion on phase diagrams, the A and B components of a binary A-B mixture will be shown on the left and right of a phase diagram, respectively, which means that the mole fraction $x_{B}^{(l)}$ will 
increase from 0 on the left to 1 on the right, while the mole fraction $x_{A}^{(l)}$ will decrease from 1 on the left to 0 on the right.

The binary systems scientists examine for VLE are generally under pressures that are not very high and at temperatures that are very low. Therefore, it is a common practice in the field to represent the data in the vapor phase using pressure without resorting to fugacity and fugacity coefficients. ${ }^{22}$ However, in the liquid phase we will be generally dealing with non-ideal solutions. Therefore, activities and activity coefficients should be adopted for the two components in the liquid phase. With activity and activity coefficient for component B defined as:

$$
\begin{aligned}
& a_{B}=\frac{p_{B}}{p_{B}^{*}} \\
& \gamma_{B}=\frac{a_{B}}{x_{B}^{(l)}}
\end{aligned}
$$

the partial pressure of component B in a binary system becomes:

$$
p_{B}=p_{B}^{*} a_{B}=p_{B}^{*} \gamma_{B} x_{B}^{(l)}
$$

As $x_{B}^{(l)} \rightarrow 0$, by relating eqn 9 with Henry's law as shown in eqn 2 where B is solute, we have

$$
\gamma_{B}(0)=\frac{K_{B}}{p_{B}^{*}}
$$

As $x_{B}^{(l)} \rightarrow 1$, by relating eqn 9 and Raoult's law as shown in eqn 3 where B is solvent, we have

$$
\gamma_{B}(1)=1
$$

By lumping $p_{B}^{*}$ and $\gamma_{B}$ into a new parameter of $h_{B}$, eqn 9 becomes:

$$
p_{B}=p_{B}^{*} \gamma_{B} x_{B}^{(l)}=h_{B} x_{B}^{(l)}
$$

which can be considered a generalization of Raoult's law in eqn 3 and Henrys' law in eqn 2, where the coefficients $p_{B}^{*}$ and $K_{B}$ for the respective cases of B as solvent and solute are now bridged by a hybrid parameter $h_{B}$, which has the activity coefficients bundled into it. Next, we will illustrate Raoult's law and Henry's law with representative experimental data available in the literature, 
calculate the activities and activity coefficients, interpret the physical meaning of the hybrid parameter $h_{B}$, and show how eqn 12 can be reduced to Raoult's law and Henry's law under limiting conditions.
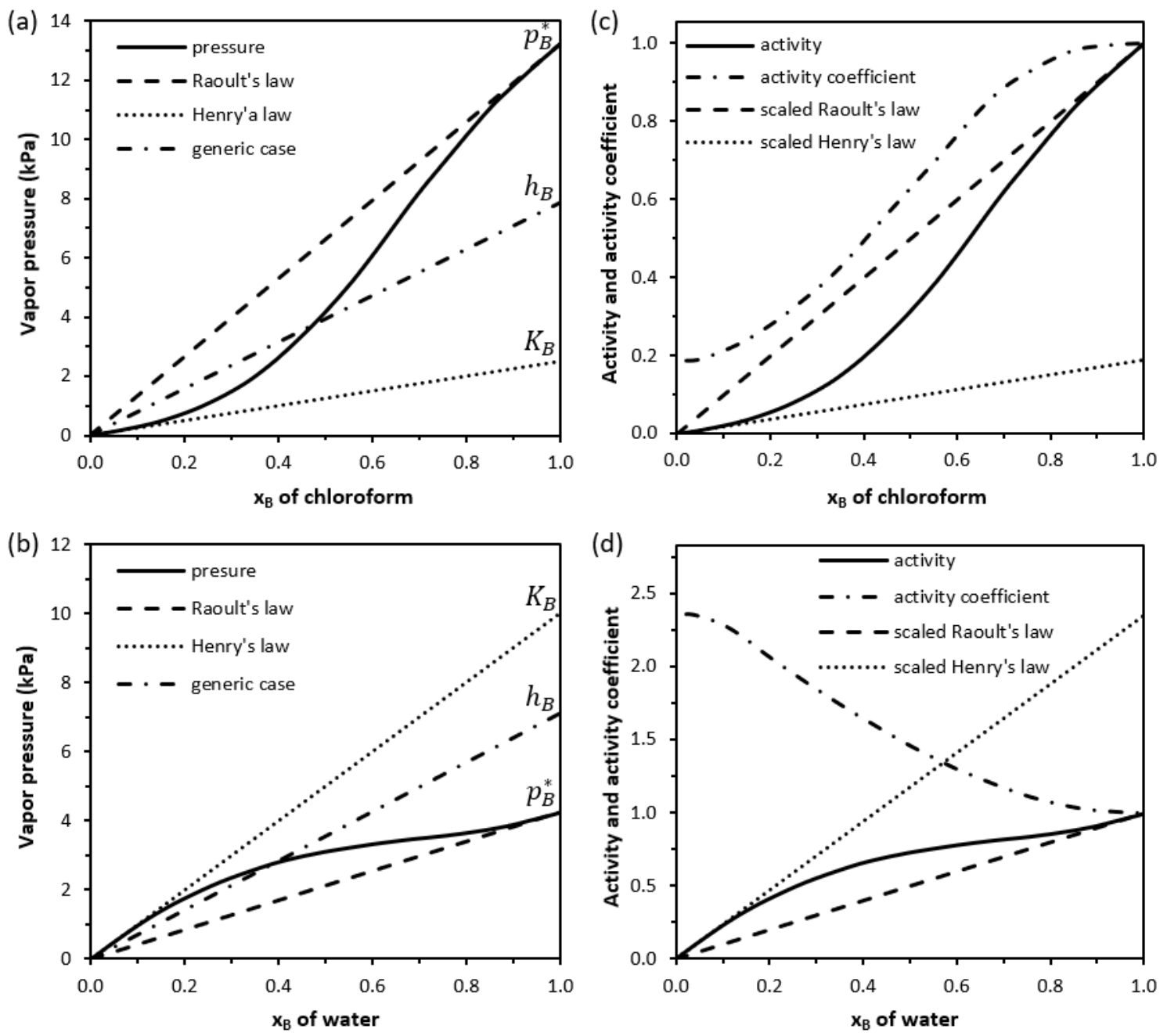

Figure 2. Partial pressures, activities, and activity coefficients of representative binary mixtures.

(a) Partial pressure of chloroform in triethylamine-chloroform $\left\{x_{A} \mathrm{~N}\left(\mathrm{C}_{2} \mathrm{H}_{5}\right)_{3}+x_{B} \mathrm{CHCl}_{3}\right\}$ at 283.15 K. (b) Partial pressure of water in ethanol-water $\left\{x_{A} \mathrm{C}_{2} \mathrm{H}_{5} \mathrm{OH}+x_{B} \mathrm{H}_{2} \mathrm{O}\right\}$ at 303.15 K. (c) and (d) are the activities and activity coefficients calculated using eqns 7 and 8 and the data in (a) and (b), respectively. Data in (a) and (b) are digitized from ref. ${ }^{23}$, respectively. For clarity, $h_{B}$ 
curves are not shown in (a) and (b) but can be scaled by a factor of $p_{B}^{*}$ based on the $\gamma_{B}$ curves in (c) and (d).

Figure $2 \mathrm{a}, \mathrm{b}$ shows the partial pressures of chloroform in the binary triethylamine-chloroform mixture and of water in the binary ethanol-water mixture as a function of their mole fractions, along with Raoult's law and Henry's law as indicated by the dashed and dotted lines, respectively. The partial pressure curves display a negative and a positive deviation from Raoult's law, as indicated by the concave and convex curvatures, respectively. As expected, Raoult's law is asymptotically approached by the partial pressure curve when $x_{B}^{(l)} \rightarrow 1$, with a tangent slope corresponding to the vapor pressure of pure component $p_{B}^{*}$, while Henry's law is asymptotically approached by the partial pressure curve when $x_{B}^{(l)} \rightarrow 0$, with a tangent slope corresponding to the Henry's law constant $K_{B}$. Note that $p_{B}^{*}>K_{B}$ (or $\left.K_{B} / p_{B}^{*}<1\right)$ in the case of negative deviation but $p_{B}^{*}<K_{B}$ (or $\left.K_{B} / p_{B}^{*}>1\right)$ in the case of positive deviation from Raoult's law. It can be further seen that the partial pressure curves in the entire range of mole fraction are bounded by the two tangent lines. If the binary mixture were an ideal solution, the concave or convex curves would collapse to a straight line that fully obeys Raoult's law in the entire range of mole fractions. This means that for an ideal solution the two tangent lines would become just one identical line with a slope of $p_{B}^{*}=K_{B}$. Conversely, the larger deviation a solution is from ideality, the larger difference is between $p_{B}^{*}$ and $K_{B}$.

Figure 2c,d shows the activities and activity coefficients calculated using eqns 7 and 8 from the partial pressures shown in Fig. 2a,b. According to eqn 7, activities are obtained by simply scaling down the partial pressures using the pure component pressure $p_{B}^{*}$ to a dimensionless value in the range of 0 to 1 . Due to the simple scaling effect, the activities in Fig. 2c,d maintain the same 
negative and positive curvatures as the partial pressures in Fig. 2a,b. Likewise, Raoult's law and Henry's law in Fig. 2a,b are also scaled down by $p_{B}^{*}$ to the dashed and dotted lines in Fig. 2c,d. The scaled Raoult's law becomes a diagonal line, because the vertical axis values are equal to the horizontal axis values, both in the ranges of $[0,1]$. According to eqn 8 , the dimensionless activity coefficients are obtained by dividing each value of the activity curve in Fig. 2 c,d by the point on the diagonal line at the same mole fraction. According to eqn 9, activity coefficients can be also obtained by dividing the partial pressure in Fig. 2a,b by the pressure on the straight line according to Raoult's law, i.e., $p_{B} /\left(p_{B}^{*} x_{B}^{(l)}\right)$. The difference between these two methods is only that the data in Fig. 2a,b are not scaled but those in Fig. 2c,d are scaled by $p_{B}^{*}$. A third method to obtain the activity coefficients is to project the activities in Fig. $2 \mathrm{c}, \mathrm{d}$ at each mole fraction from the origin to the right axis at $x_{B}^{(l)}=1$. All these different ways lead to the same activity coefficients with a monotonical upward or downward trend as a function of mole fractions. On the left end of $x_{B}^{(l)} \rightarrow$ $0, \gamma_{B}$ approaches a constant value of $\gamma_{B}(0)=0.19$ or 2.36 that can be calculated using eqn 10 , with the value being smaller or larger than 1 depending on the negative and positive deviations. However, on the right end of $x_{B}^{(l)} \rightarrow 1, \gamma_{B}$ approaches only one identical constant $\gamma_{B}(1)=1$ as indicated by eqn 11, regardless of the negative or positive deviations. The reason activity coefficients approach constant values of $\gamma_{B}(0)$ and $\gamma_{B}(1)$ is that the partial pressure curve can be well described by linear equations of Henry's law and Raoult's law as $x_{B}^{(l)}$ approaches 0 and 1 . It can be expected that in case of ideal solution, the value of $\gamma_{B}$ would remain as 1 in the entire range of mole fractions. Therefore, the deviation of $\gamma_{B}$ from the value of 1 is an indication of the nonideality of a solution at $x_{B}^{(l)}$ values not too close to 1 . In fact, by using the $\gamma_{B}(0)$ values, the intermolecular interactions between $\mathrm{A}$ and $\mathrm{B}$ components relative to the average interactions 
among pure $\mathrm{A}$ and pure $\mathrm{B}$ can be quantitatively calculated as $\Delta E=\beta k_{B} T=\ln \gamma_{B}(0) k_{B} T=-1.66$ $k_{B} T$ or $+0.86 k_{B} T$ based on statistical mechanics understandings (Supplementary Materials). These negative and positive $\Delta E$ values indicate an attraction or repulsion between $\mathrm{A}$ and $\mathrm{B}$ components compared to their pure counterparts, which are the fundamental reasons for solutions to display negative and positive deviations from the Raoult's law. ${ }^{13}$

Further comparing Fig. 2a,b and Fig. 2c,d, it is straightforward to see that these figures are essentially the same, except for the scaling factor of $p_{B}^{*}$. This makes it convenient to understand the physical meaning of $h_{B}$. According to the definition of $p_{B}^{*} \gamma_{B}$ shown in eqn 12 , the hybrid parameter $h_{B}$ has a dimension of pressure, and its curves (not shown) can be obtained by scaling up the activity coefficients $\gamma_{B}$ in Fig. 2c,d using the pure component pressure $p_{B}^{*}$. Consequently, the $h_{B}$ curves should maintain exactly the same upward or downward trend as the activity coefficients in Fig. 2c,d. Additionally, the $h_{B}$ curves can be also obtained by simply projecting the partial pressures in Fig. 2a,b at each mole fraction from the origin to the right axis at $x_{B}^{(l)}=1$, in the same way that activity coefficients are obtained from activities in Fig. 2c,d by the third method mentioned above. Therefore, the $h_{B}$ values are fully enclosed in an interval of $\left[K_{B}, p_{B}^{*}\right]$ in the case of negative deviation and in an interval of $\left[p_{B}^{*}, K_{B}\right]$ in the case of positive deviation from Raoult's law. Only when the mole fraction $x_{B}^{(l)}$ approaches 1 and 0 , the hybrid parameter $h_{B}$ asymptotically approaches the limiting values of $p_{B}^{*}$ and $K_{B}$. Like $\gamma_{B}$, the deviation of $h_{B}$ from the value of $p_{B}^{*}$ is an indication of the non-ideality of a solution at $x_{B}^{(l)}$ values not too close to 1 . Recently Yin interpreted $p_{B}^{*}$ and $K_{B}$ as the saturation vapor pressure and the apparent saturation vapor pressure of component B as solvent and solute at $x_{B}^{(l)} \rightarrow 1$ and 0 , respectively, and a projected $K_{B}^{\prime}$, which is similar to the $h_{B}$ defined here in terms of activity coefficients, as the apparent saturation vapor 
pressure of component $\mathrm{B}$ in the non-dilute solution region of $x_{B}^{(l)} \in(0,1) .{ }^{24}$ Further explanations for the physical meanings of activity $\alpha_{B}$, activity coefficient $\gamma_{B}$, and the hybrid parameter $h_{B}$ in terms of intermolecular interactions $\Delta E=\beta k_{B} T$ from the standpoint of statistical mechanics can be found in the Supplementary Materials.

Finally, it is straightforward to show that under the limiting conditions of $x_{B}^{(l)} \rightarrow 1$ and 0 , eqn 12 can be reduced back to Raoult's law and Henry's law as shown by:

$$
\begin{aligned}
& p_{B}=h_{B}(1) x_{B}^{(l)}=p_{B}^{*} \gamma_{B}(1) x_{B}^{(l)}=p_{B}^{*} x_{B}^{(l)} \\
& p_{B}=h_{B}(0) x_{B}^{(l)}=p_{B}^{*} \gamma_{B}(0) x_{B}^{(l)}=K_{B} x_{B}^{(l)}
\end{aligned}
$$

This indicates that eqn 12 can be understood as a generalization of Raoult's law and Henry's law. Next, we will make further simple derivations based on eqn 12 to show that it is a useful model for qualitative and semi-quantitative interpretations of binary solutions forming negative and positive azeotropes.

3.2. Overlapping rule of $\boldsymbol{h}_{\boldsymbol{A}}$ and $\boldsymbol{h}_{\boldsymbol{B}}$ ranges. Binary mixtures typically consist of two components with different vapor pressures $p_{A}^{*} \neq p_{B}^{*}$. For the convenience of discussions in this section, let us set $p_{B}^{*}>p_{A}^{*}$ and put component $\mathrm{A}$ on the left and component $\mathrm{B}$ on the right of the phase diagram. Regardless of non-azeotropic or azeotropic mixtures, the total pressure on the left end of phase diagram where $x_{B}^{(l)}$ is close to 0 is mainly contributed by solvent $\mathrm{A}$ and slightly by solute B. Following eqns 5, 6, and 12-14, the total pressure is:

$$
p=p_{A}^{*} x_{A}^{(l)}+K_{B} x_{B}^{(l)}=p_{A}^{*}\left(1-x_{B}^{(l)}\right)+K_{B} x_{B}^{(l)}=p_{A}^{*}+\left(K_{B}-p_{A}^{*}\right) x_{B}^{(l)}
$$

Therefore, the tangent line for the total pressure curve at $x_{B}^{(l)}$ close to 0 has an intercept at $p_{A}^{*}$ on the left vertical axis and a slope of $K_{B}-p_{A}^{*}$. Likewise, the total pressure on the right end of phase 
diagram where $x_{B}^{(l)}$ is close to 1 is mainly contributed by solvent B and slightly by solute A. Following eqns 5, 6 and 12-14, the total pressure is:

$$
p=p_{B}^{*} x_{B}^{(l)}+K_{A} x_{A}^{(l)}=p_{B}^{*} x_{B}^{(l)}+K_{A}\left(1-x_{B}^{(l)}\right)=K_{A}+\left(p_{B}^{*}-K_{A}\right) x_{B}^{(l)}
$$

Therefore, the tangent line for the total pressure curve at $x_{B}^{(l)}$ close to 1 has an intercept at $K_{A}$ on the left vertical axis and a slope of $p_{B}^{*}-K_{A}$. As shown above in Fig. $2 \mathrm{a}$, we have $p_{A}^{*}>K_{A}$ and $p_{B}^{*}>$ $K_{B}$ for a binary system with negative deviations. Since we set $p_{B}^{*}>p_{A}^{*}$, it must be true that $p_{B}^{*}>$ $K_{A}$. According to eqn 16, the second tangent line must have a positive slope. As for the first tangent line, the slope could be positive if $p_{A}^{*}<K_{B}$ or negative if $p_{A}^{*}>K_{B}$. In comparison, as shown above in Fig. 2b, we have $p_{A}^{*}<K_{A}$ and $p_{B}^{*}<K_{B}$ for a binary system with positive deviations. Since we set $p_{B}^{*}>p_{A}^{*}$, it must be true that $p_{A}^{*}<K_{B}$. According to eqn 15 , the first tangent line must have a positive slope. As for the second tangent line, the slope is positive if $p_{B}^{*}>K_{A}$ and negative if $p_{B}^{*}<$ $K_{A}$

For a binary mixture to form an azeotrope, the slopes of the two tangent lines must have opposite signs, so that a minimum or a maximum point can be formed. As noted in the methodology section, we exclude the scenarios with more than one extremum point. Therefore, for a binary system with negative deviations, both conditions of $p_{A}^{*}>K_{B}$ and $p_{B}^{*}>K_{A}$ must be satisfied, which leads to a concave total pressure curve connecting $p_{A}^{*}$ and $p_{B}^{*}$. In contrast, for a binary system with positive deviations, both conditions of $p_{A}^{*}<K_{B}$ and $p_{B}^{*}<K_{A}$ must be satisfied, which leads to a convex total pressure curve connecting $p_{A}^{*}$ and $p_{B}^{*}$. In other words, to form an azeotrope the $h_{A}$ range of $\left[K_{A}, p_{A}^{*}\right]$ must overlap with the $h_{B}$ range of $\left[K_{B}, p_{B}^{*}\right]$ in the case of negative deviations, or the $h_{A}$ range of $\left[p_{A}^{*}, K_{A}\right]$ must overlap with the $h_{B}$ range of $\left[p_{B}^{*}, K_{B}\right]$ in the case of positive deviations. For a scenario with $p_{B}^{*}>p_{A}^{*}$, the condition of $p_{A}^{*}>K_{B}$ for a binary solution with negative deviation simply means that the negative deviation of component B needs 
to be large enough so that $K_{B}$ drops under $p_{A}^{*}$; and likewise, the condition of $p_{B}^{*}<K_{A}$ for a binary solution with positive deviation simply means that the positive deviation of component A needs to be large enough so that $K_{A}$ rises above $p_{B}^{*}$. By using eqn 10 and the statistical mechanics eqns s1s4, these conditions simply indicate that the intermolecular interactions between the two components $\mathrm{A}-\mathrm{B}$ should be stronger than $\left|\ln \left(p_{A}^{*} / p_{B}^{*}\right)\right| k_{B} T$ (Supplementary Materials). If the difference between $p_{A}^{*}$ and $p_{B}^{*}$ is small, the deviation from Raoult's law does not need to be large for binary mixtures to form azeotropes.

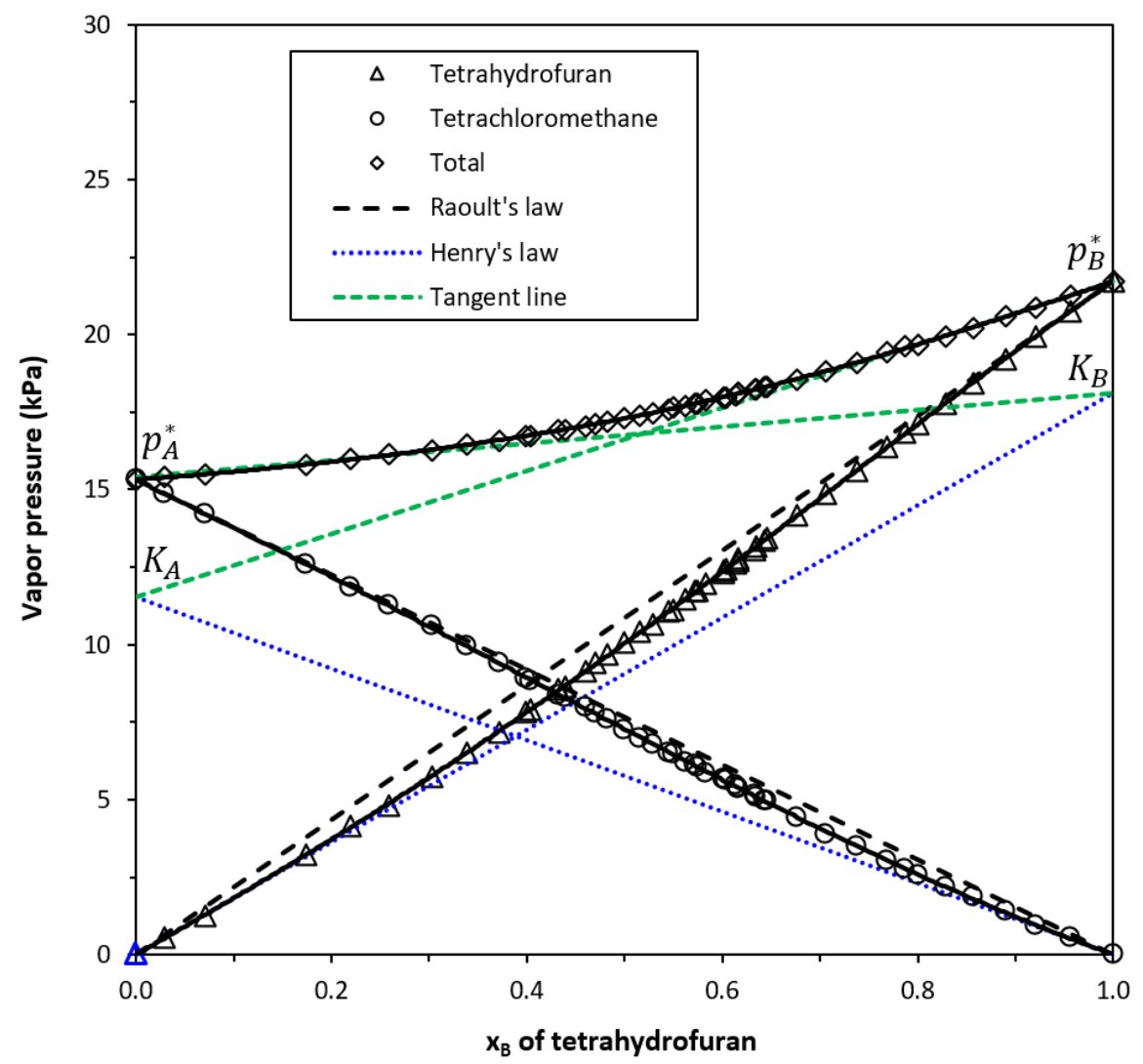

Figure 3. Data analysis for the binary non-azeotropic tetrachloromethane-tetrahydrofuran $\left\{x_{A} \mathrm{CCl}_{4}+x_{B} \mathrm{C}_{4} \mathrm{H}_{8} \mathrm{O}\right\}$ with negative deviation from Raoult's law at $298.15 \mathrm{~K}$ (data taken from refs. $\left.{ }^{10,25}\right)$. The values of Henry's law constant $K_{A}$ and $K_{B}$ are calculated by the averaged slopes of 
Henry's law and the tangent line on the right and left sides, respectively. For clarity, the vapor compositions are not shown.

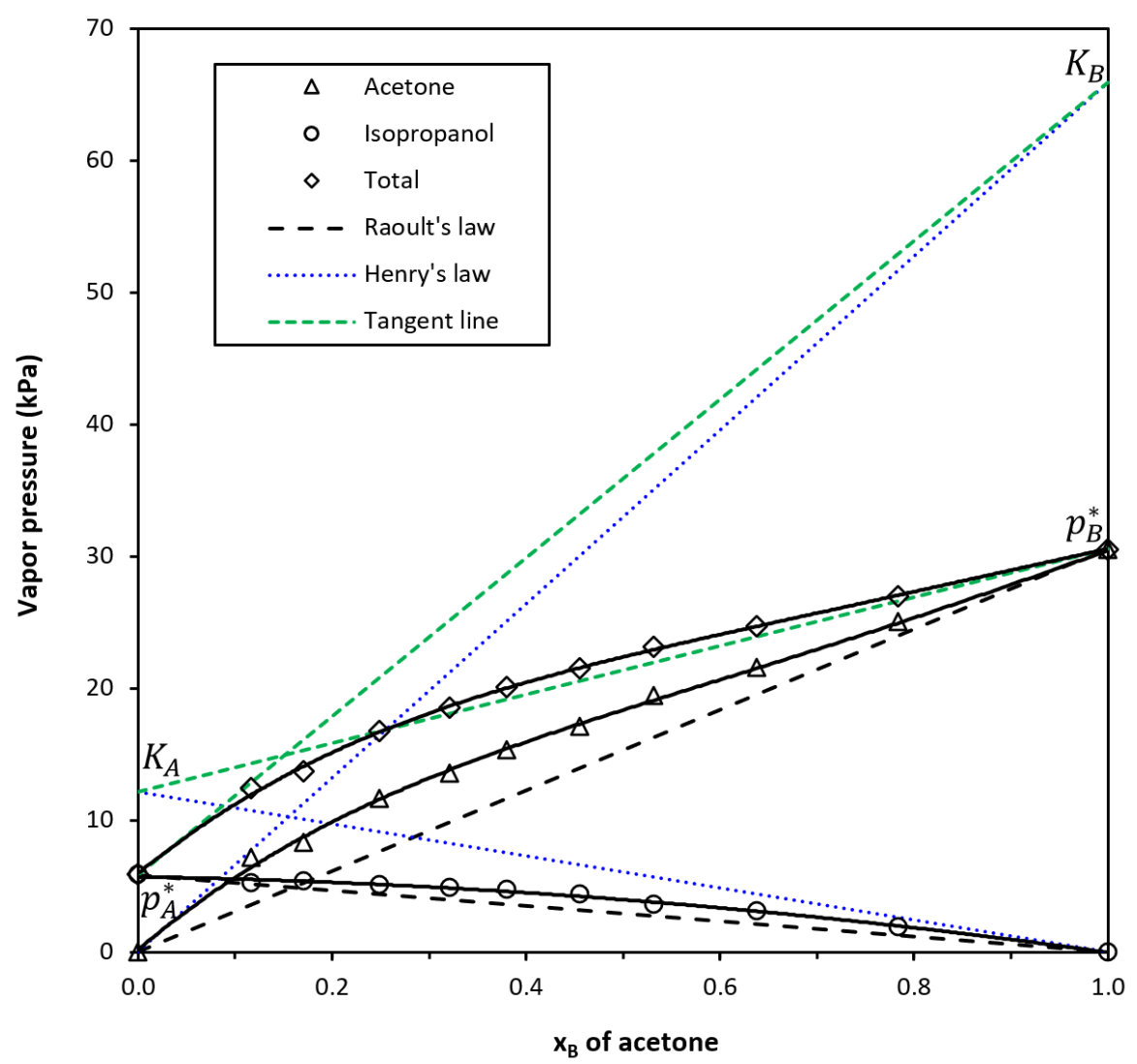

Figure 4. Data analysis for the binary non-azeotropic isopropanol-acetone mixture $\left\{x_{A} \mathrm{C}_{3} \mathrm{H}_{8} \mathrm{O}+\right.$ $x_{B} \mathrm{C}_{3} \mathrm{H}_{6} \mathrm{O}$ \} with positive deviation from Raoult's law at $298.15 \mathrm{~K}$ (data taken from ref. ${ }^{11}$ ). The values of Henry's law constant $K_{A}$ and $K_{B}$ are calculated by the averaged slopes of Henry's law and the tangent line on the right and left sides, respectively. For clarity, the vapor compositions are not shown.

Without overlapping $h_{A}$ and $h_{B}$ ranges, the mixture will be a non-azeotrope instead of an azeotrope. We illustrate the absence of overlapping $h_{A}$ and $h_{B}$ ranges in non-azeotropic binary mixtures with two representative examples. Figures 3 and 4 show the data analyses for 
tetrachloromethane-tetrahydrofuran and isopropanol-acetone binary mixtures previously shown in Fig. 1a,c, with negative and positive deviations from Raoult's law, respectively. For the tetrachloromethane-tetrahydrofuran mixture with negative deviation, the $h_{A}$ range of $\left[K_{A}, p_{A}^{*}\right]$ and the $h_{B}$ range of $\left[K_{B}, p_{B}^{*}\right]$ do not overlap, as can be seen from the order of $K_{A}<p_{A}^{*}<K_{B}<p_{B}^{*}$. As mentioned above, an azeotrope would be formed if the negative deviation of component B could be slightly enlarged so that $K_{B}$ drops under $p_{A}^{*}$. Likewise, for the isopropanol-acetone mixture with positive deviation, the $h_{A}$ range of $\left[p_{A}^{*}, K_{A}\right]$ and the $h_{B}$ range of $\left[p_{B}^{*}, K_{B}\right]$ do not overlap either, as can be seen from the order of $p_{A}^{*}<K_{A}<p_{B}^{*}<K_{B}$. As mentioned above, an azeotrope would be formed if the positive deviation of component A could be slightly enlarged so that $K_{A}$ rises above $p_{B}^{*}$. Consequently, the two tangent lines represented by eqns 15 and 16 have positive slopes in both Figs. 3 and 4, and therefore no azeotropes can be formed in these two cases. For ideal solutions with $p_{A}^{*}=K_{A}$ and $p_{B}^{*}=K_{B}$, the $h_{A}$ and $h_{B}$ ranges are condensed to just two points for the two components. Binary mixtures typically consist of two components with different vapor pressures $p_{A}^{*} \neq p_{B}^{*}$, and therefore, ideal solutions do not form azeotropes due to lack of overlap of $h_{A}$ and $h_{B}$ ranges. In other words, azeotropes are special cases of only non-ideal, instead of ideal solutions.

3.3. Mole fraction and pressure of azeotropes. Next, we turn to the azeotropic cases with overlapping $h_{A}$ and $h_{B}$ ranges. Note that eqns 15 and 16 are only applicable for the two ends of phase diagrams, i.e., $x_{B} \approx 0$ (or $x_{A} \approx 1$ ) on the left and $x_{B} \approx 1$ (or $x_{A} \approx 0$ ) on the right. For the middle section of mole fractions, we can exploit the new parameter $h_{B}$ and the generalized eqn 12 to rationalize azeotropic compositions. Following eqns 5, 6, and 12, the total pressure is:

$$
p=p_{A}+p_{B}=h_{A} x_{A}^{(l)}+h_{B} x_{B}^{(l)}=h_{A}\left(1-x_{B}^{(l)}\right)+h_{B} x_{B}^{(l)}=h_{A}+\left(h_{B}-h_{A}\right) x_{B}^{(l)}
$$

For azeotropes that display a single minimum or maximum in total pressure, the derivative of total 
pressure $p$ should be zero at the minimum or the maximum. In the next section, we will show that the minimum or the maximum exactly corresponds to the negative or positive azeotrope. For the extremum point, we have:

$$
\frac{d p}{d x_{B}^{(l)}}=h_{B}-h_{A}=0
$$

which leads to:

$$
h_{A}=h_{B}=h
$$

The same conclusion was obtained by Yin recently using projected $K_{B}^{\prime}$ similar to the $h_{B}$ defined in this work. ${ }^{24}$ However, the derivation started from a known conclusion of $n_{A}^{(l)} / n_{B}^{(l)}=n_{A}^{(g)} / n_{B}^{(g)}$ rather than a starting condition for azeotropes. ${ }^{26}$ Eqn 19 means that an azeotrope is obtained when the $h_{B}$ for component $\mathrm{B}$ at a mole fraction of $x_{B}^{(l)}$ is equal to the $h_{A}$ for component $\mathrm{A}$ at the corresponding mole fraction of $x_{A}^{(l)}$ (or 1- $x_{B}^{(l)}$ ). According to eqns 6 and 17, the derivatives of the partial pressures $p_{A}$ and $p_{B}$ at the azeotropic mole fraction must be of opposite sign but of the same magnitude so that eqn 18 can be satisfied. In addition, according to eqns 5, 6, 12, and 19, the total pressure for the system is:

$$
p=h_{A} x_{A}^{(l)}+h_{B} x_{B}^{(l)}=h\left(x_{A}^{(l)}+x_{B}^{(l)}\right)=h \cdot 1=h
$$

This means that the total pressure at the azeotrope composition is the common $h$. Now it becomes clear why the condition of overlapping $h_{A}$ and $h_{B}$ ranges identified above must be satisfied for a binary mixture to form an azeotrope. Only under this condition it is possible to have a common $h$, which does not only dictate the azeotrope composition, but is also equal to the total pressure of the azeotropic system, regardless of negative or positive deviations.

Next, we illustrate these understandings using available experimental data for a few azeotropes with both negative and positive deviations. Figures 5 and 6 show the data analyses for the 
acetone-chloroform and acetone-carbon disulfide binary mixtures previously shown in Fig. 1b,d. For the acetone-chloroform system with negative deviations, we can see the order of $p_{A}^{*}>p_{B}^{*}>$ $K_{A}>K_{B}$. Therefore, the $h_{A}$ range of $\left[K_{A}, p_{A}^{*}\right]$ overlaps with the $h_{B}$ range of $\left[K_{B}, p_{B}^{*}\right]$. Additionally, the two tangent lines of the total pressure curve have opposite signs, leading to a minimum azeotropic point on the concave total pressure curve. In contrast, for the acetone-carbon disulfide system with positive deviations, we can see the order of $p_{A}^{*}<p_{B}^{*}<K_{B}<K_{A}$. Therefore, the $h_{A}$ range of $\left[p_{A}^{*}, K_{A}\right]$ overlaps with the $h_{B}$ range of $\left[p_{B}^{*}, K_{B}\right]$. Additionally, the two tangent lines of the total pressure curve have opposite signs, in agreement with the presence of a maximum azeotropic point on the convex total pressure curve.

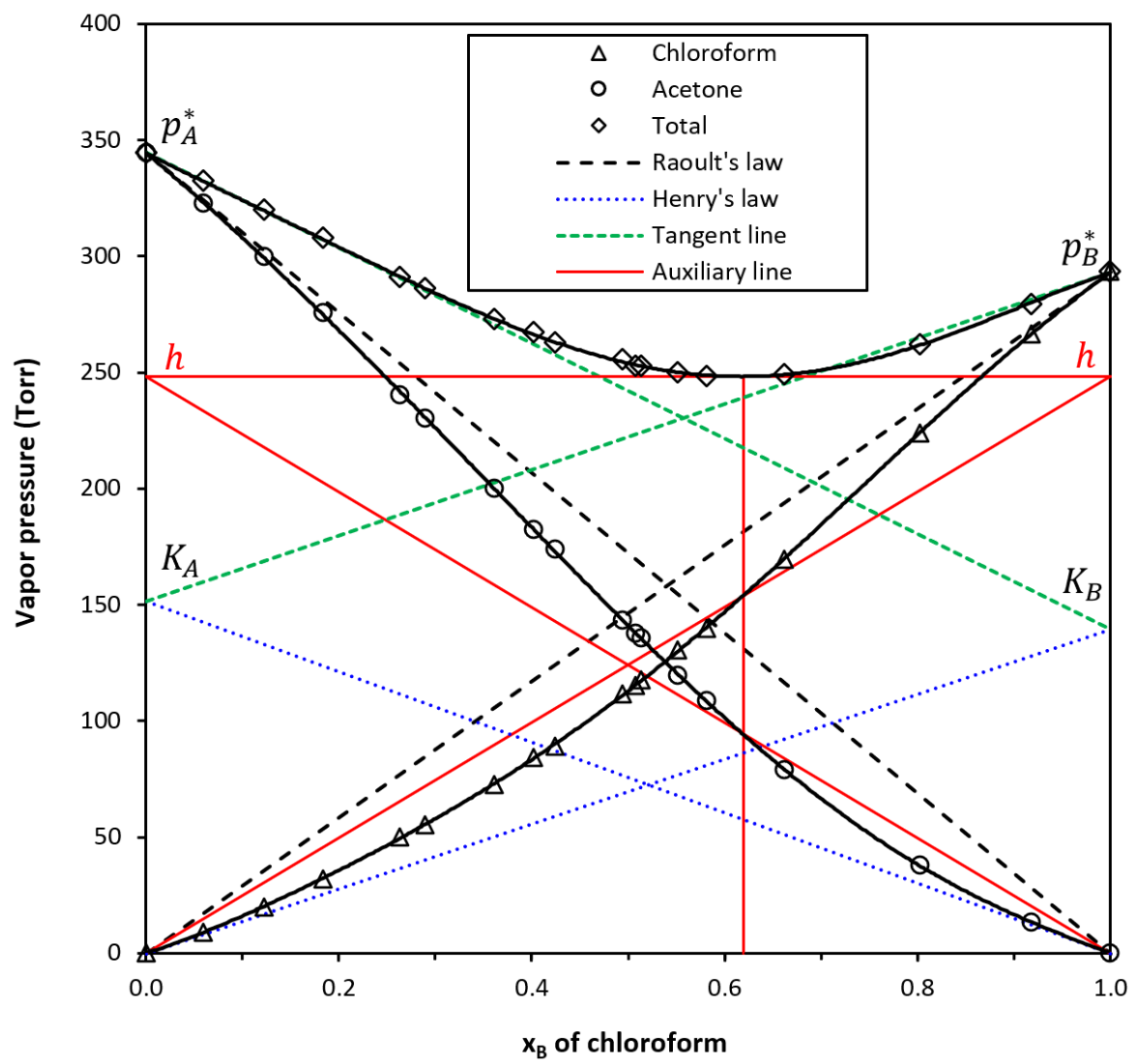

Figure 5. Data analysis for the binary mixture of acetone-chloroform $\left\{x_{A} \mathrm{C}_{3} \mathrm{H}_{6} \mathrm{O}+x_{B} \mathrm{CHCl}_{3}\right\}$ forming negative azeotrope at $308.32 \mathrm{~K}$ (data taken from ref. ${ }^{12}$ ). The values of Henry's law 
constant $K_{A}$ and $K_{B}$ are calculated by the averaged slopes of Henry's law and the tangent line on the right and left sides, respectively, and agree with the experimental values in refs. ${ }^{22,27}$. For clarity, the vapor compositions are not shown.

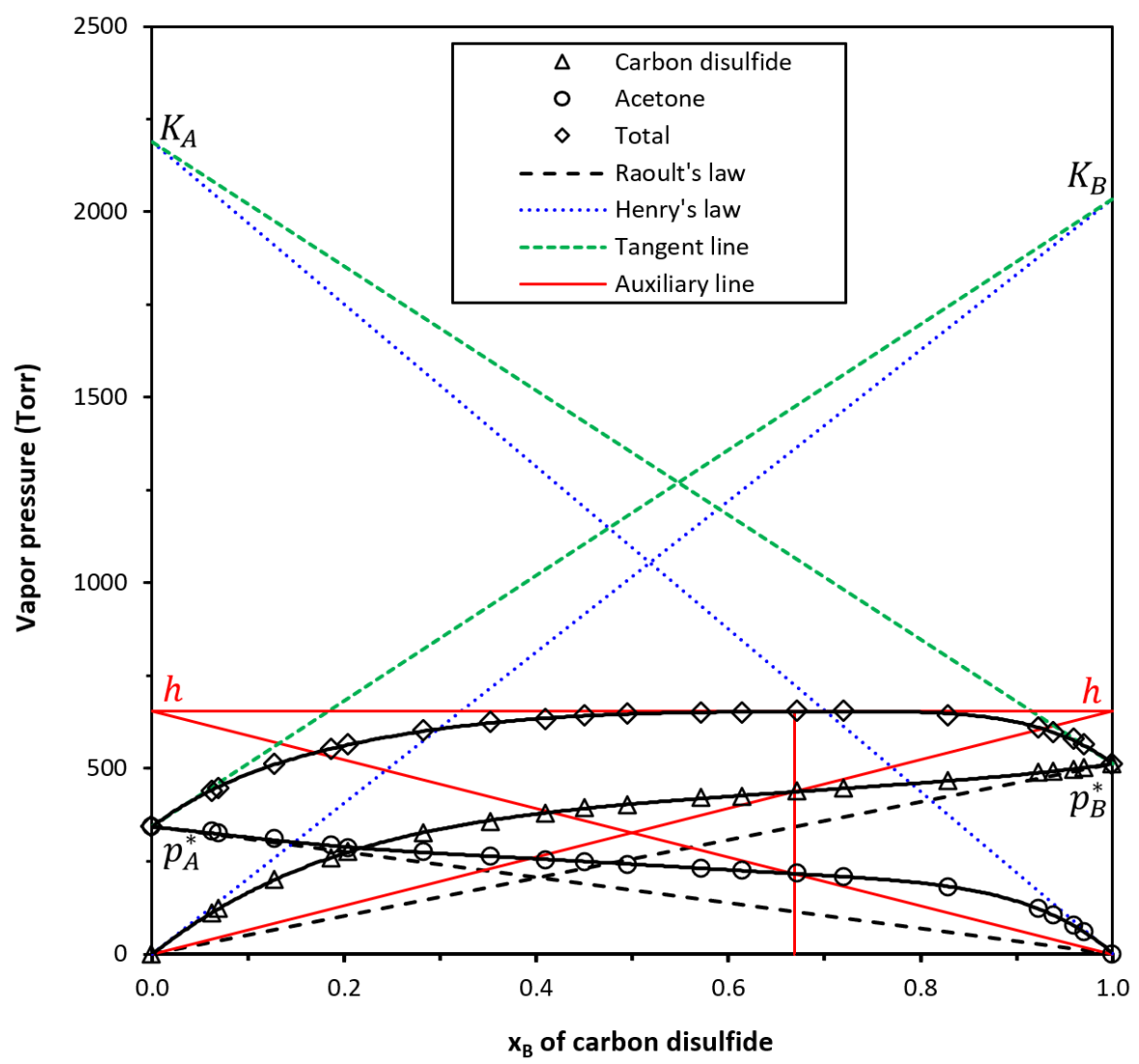

Figure 6. Data analysis for the binary mixture of acetone-carbon disulfide $\left\{x_{A} \mathrm{C}_{3} \mathrm{H}_{6} \mathrm{O}+x_{B} \mathrm{CS}_{2}\right\}$ forming positive azeotrope at $308.32 \mathrm{~K}$ (data taken from ref. ${ }^{12}$ ). The values of Henry's law constant $K_{A}$ and $K_{B}$ are calculated by the averaged slopes of Henry's law and the tangent line on the right and left, respectively. For clarity, the vapor compositions are not shown.

Figures 5 and 6 further show our data analysis following eqns 19 and 20. One can first draw an auxiliary horizontal line tangent with the concave or convex total pressure curve at the minimal or maximal pressure for the cases with negative or positive deviations. The tangent points should 
be located exactly at the azeotropic points. This horizontal line intersects with the two vertical axes at $h_{A}$ on the left and $h_{B}$ on the right, which is equal to the common pressure $h=248.4$ Torr for the azeotrope. One can then draw an auxiliary vertical line from the azeotropic point to find the mole fractions $x_{B}=0.62$ for the azeotropes. According to our discussions associated with Fig. 2, the $h_{A}$ point on the left vertical axis can be obtained by projecting the partial pressure on the $p_{A}$ curve at the azeotropic composition of $x_{A}=1-x_{B}=0.38$ from point $\mathrm{B}\left(x_{A}^{(l)}=0\right)$ to the left vertical axis $\left(x_{A}^{(l)}=1\right)$, and meanwhile the $h_{B}$ point on the right vertical axis can be obtained by projecting the partial pressure on the $p_{B}$ curve at the azeotropic composition of $x_{B}=0.62$ from point $\mathrm{A}\left(x_{B}^{(l)}=\right.$ $0)$ to the right vertical axis $\left(x_{B}^{(l)}=1\right)$. These expectations are indeed confirmed to be the case for both systems in Figs. 5 and 6. Alternatively, the auxiliary lines shown in Figs. 5 and 6 can be drawn in a different order. After obtaining $h_{A}$ and $h_{B}$ by drawing a horizontal tangent line, one can then connect point $h_{A}$ with point $\mathrm{B}$, and connect point $h_{B}$ with point A. These lines will intersect with the $p_{A}$ and $p_{B}$ curves at the azeotropic mole fraction of $x_{B}=0.62$. Finally, the vertical line can be drawn at the azeotropic mole fraction to intersect with the horizontal tangent line to find the azeotropic point with $x_{B}=0.62$ and $h=248.4$ Torr. The second approach is especially useful to find the azeotropic point with $x_{B}=0.67$ and $h=655.0$ Torr for the binary system in Fig. 6 , where the total pressure near the azeotropic point shows a small variance in a wide range of mole fractions.

Figure 7 shows the first-order derivatives of the partial and total pressure curves and the calculated values of $h_{A}$ and $h_{B}$ as a function of mole fraction for the acetone-chloroform binary mixture shown in Fig. 5. To obtain smooth curves for derivatives, the experimental data points for the partial and total pressure are first fitted by polynomials of order 6 and then the analytical formulae for the first-order derivatives are obtained. Both the polynomials without constraints in 


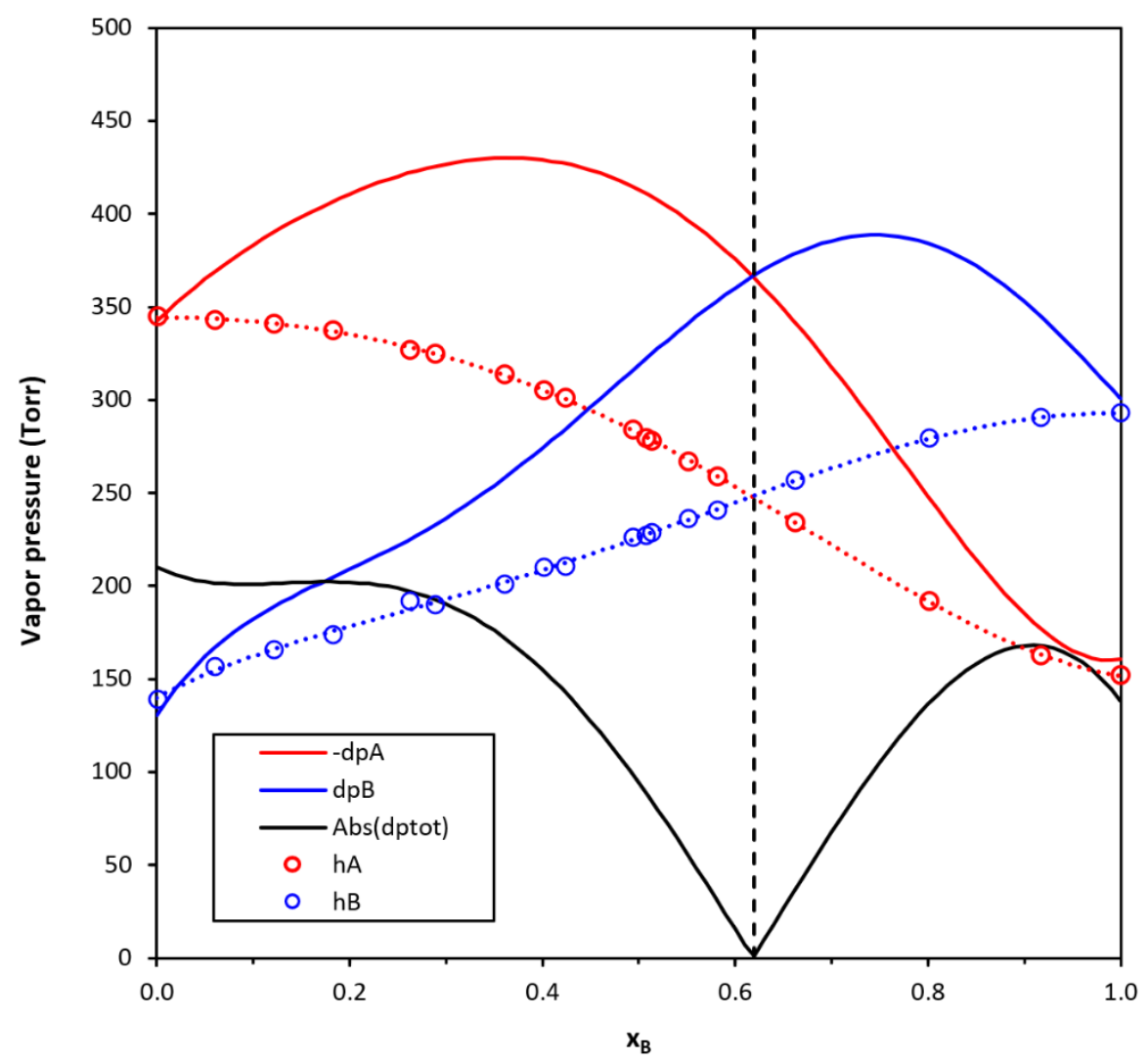

Figure 7. First-order derivatives of the partial and total pressure curves and the calculated values of $h_{A}$ and $h_{B}$ as a function of mole fraction for the binary mixture of acetone-chloroform $\left\{x_{A} \mathrm{C}_{3} \mathrm{H}_{6} \mathrm{O}+x_{B} \mathrm{CHCl}_{3}\right\}$ forming negative azeotrope at $308.32 \mathrm{~K}$ (data taken from ref. ${ }^{12}$ ). Vertical dashed line indicates the azeotropic mole fraction.

the generic form of $y=a x^{6}+b x^{5}+c x^{4}+d x^{3}+e x^{2}+f x+g$ and with constraints at the two ends of $x=0$ and 1 in the form of $y=y_{1}(1-x)+y_{2} x+\left(a x^{5}+b x^{4}+c x^{3}+d x^{2}+e x\right)(1-$ $x$ ) led to comparable results. However, the fitting quality is limited near the two ends due to small number of available data points. Consequently, as $x_{B}^{(l)}$ approaches 0 and 1 , the $-d p_{A} / d x_{B}^{(l)}$ curve and the $d p_{B} / d x_{B}^{(l)}$ curve may not fully converge with the $h_{A}$ and $h_{B}$ curves as they are supposed to. Nevertheless, at the azeotropic mole fraction of $x_{B}=0.62$, the derivatives of the partial 
pressures $p_{A}$ and $p_{B}$ are of opposite sign but of the same magnitude, leading to a zero derivative for the total pressure curve. In addition, it can be also seen that for this binary azeotropic system with negative deviation, the values of $h_{A}$ at any one $x_{B}^{(l)}$ is greater or less than the value of $h_{B}$ on the left and right of the azeotropic point, respectively; only at the azeotropic composition of $x_{B}=$ $0.62, h_{A}$ is equal to $h_{B}$ and meanwhile both are equal to the total pressure $h=248.4$ Torr of the azeotrope. It can be readily seen from Fig. 6 that the trend is exactly the opposite for the acetone-carbon disulfide binary azeotropic system with positive deviation, except at the mole fraction for the azeotrope.

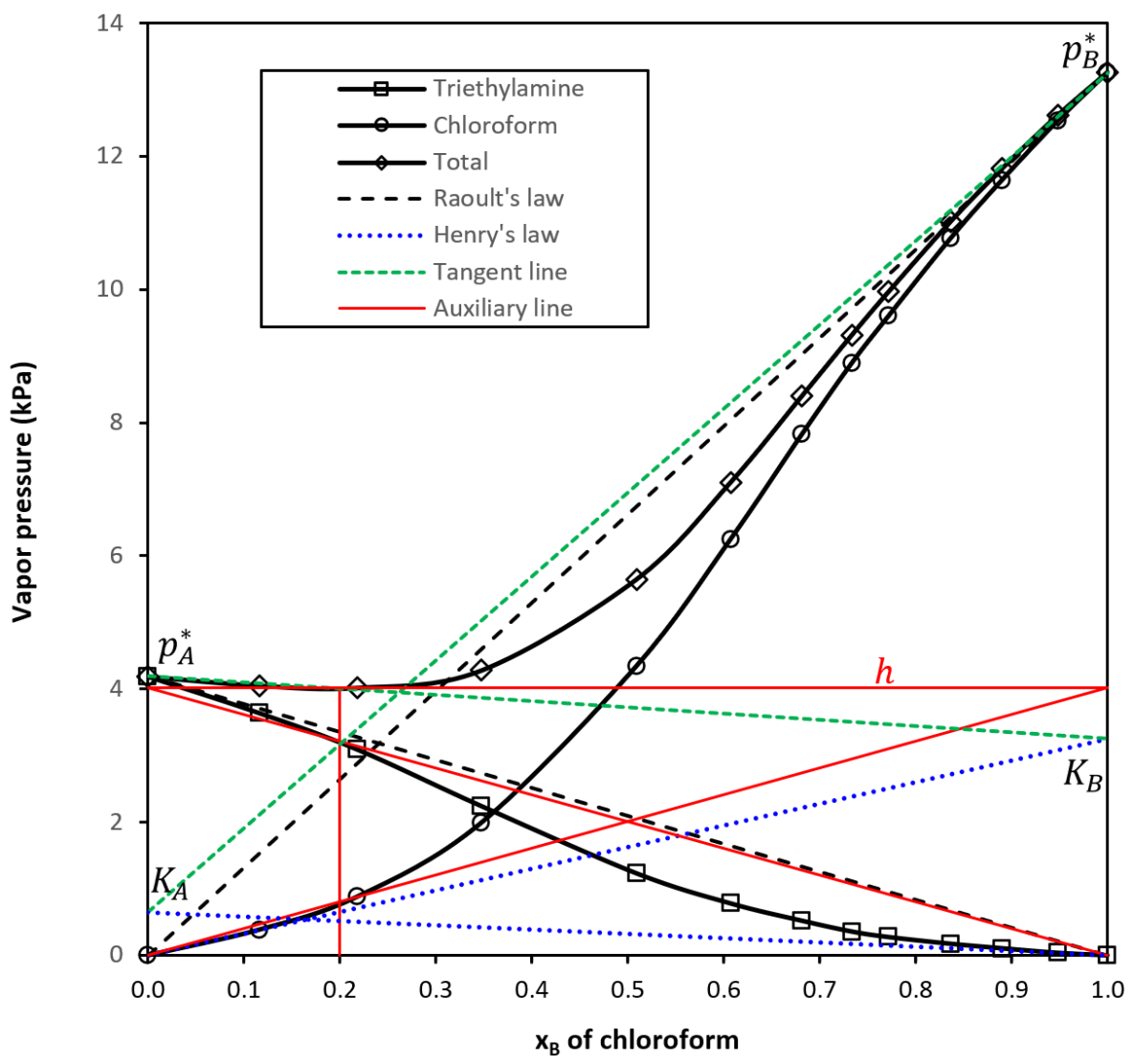

Figure 8. Data analysis for the binary mixture of triethylamine-chloroform $\left\{x_{A} \mathrm{~N}\left(\mathrm{C}_{2} \mathrm{H}_{5}\right)_{3}+\right.$ $x_{B} \mathrm{CHCl}_{3}$ \} forming negative azeotrope at $283.15 \mathrm{~K}$ (data taken from ref. ${ }^{28}$ ). The values of Henry's 
law constant $K_{A}$ and $K_{B}$ are calculated by the averaged slopes of Henry's law and the tangent line on the right and left, respectively. For clarity, the vapor compositions are not shown.

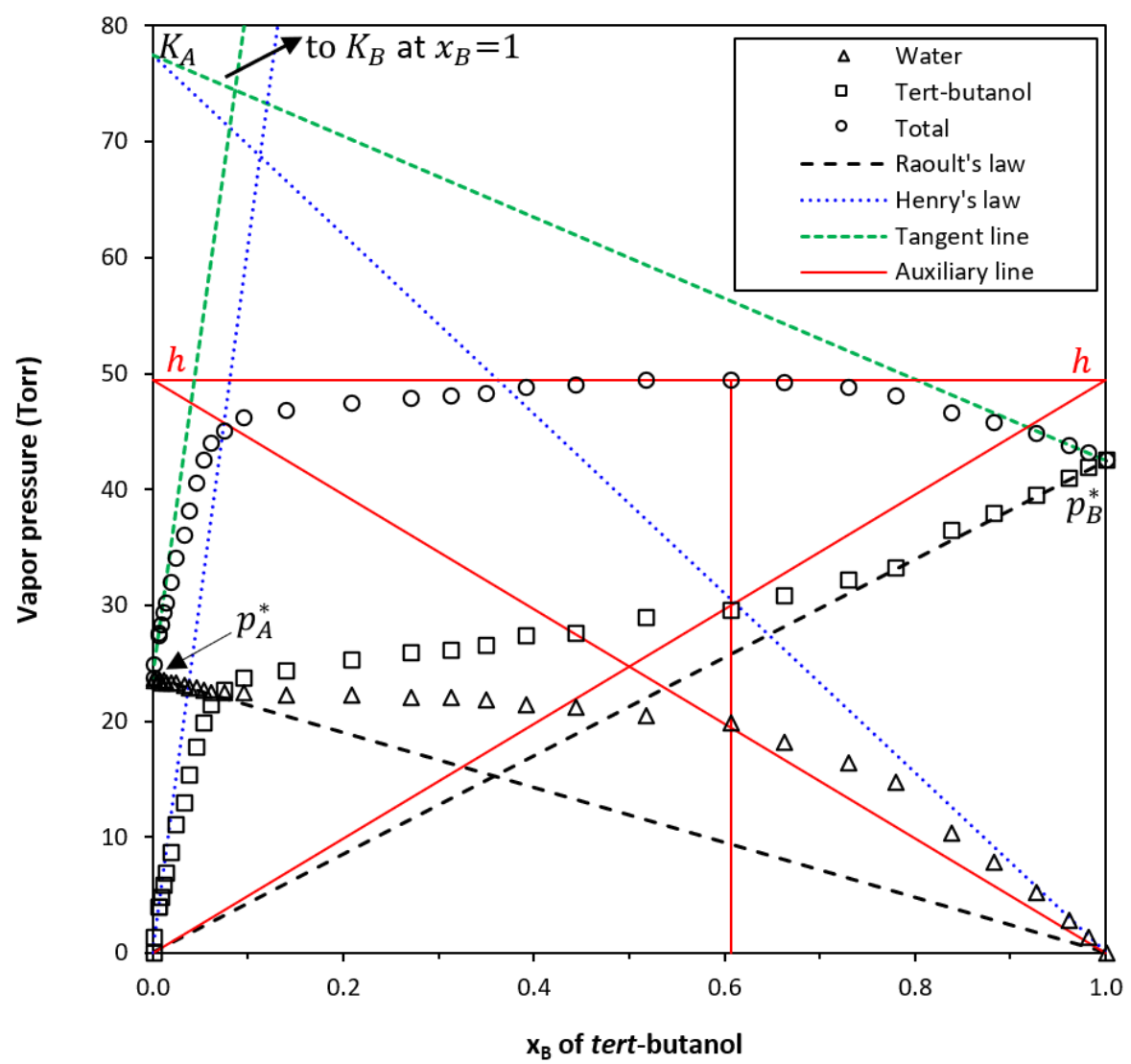

Figure 9. Data analysis for the binary mixture of water-tert-butanol $\left\{x_{A} \mathrm{H}_{2} \mathrm{O}+x_{B} \mathrm{C}_{4} \mathrm{H}_{10} \mathrm{O}\right\}$ forming positive azeotrope at $298.15 \mathrm{~K}$ (data taken ref. ${ }^{29,30}$ ). The values of Henry's law constant $K_{A}$ and $K_{B}$ are calculated by the averaged slopes of Henry's law and the tangent line on the right and left, respectively. Due to the very large Henry's law constant for tert-butanol and the very large slope of the tangent line on the left, the $K_{B}$ is not shown but only indicated by an arrow. For clarity, the vapor compositions are not shown.

To rule out the possibility that the data analysis shown in Figs. 5 and 6 is only fortuitous, we also checked two more binary azeotropic systems with negative and positive deviations. Figure 8 
shows the phase diagram for the binary mixture of triethylamine-chloroform with negative deviations. This binary system shows an order of $p_{B}^{*}>p_{A}^{*}>K_{B}>K_{A}$, which indicates an overlap of $h_{A}$ and $h_{B}$ ranges. However, unlike the binary mixture of acetone-chloroform shown in Fig. 5, here the $h_{A}$ range of $\left[K_{A}, p_{A}^{*}\right]$ overlap only marginally with the $h_{B}$ range of $\left[K_{B}, p_{B}^{*}\right]$, because $p_{A}^{*}$ is only slightly larger than $K_{B}$ although $p_{B}^{*}$ is much larger than $K_{A}$. Another difference of the current system from the previous one is that here the tangent slope at $x_{B}^{(l)}$ close to 0 is very small while the other tangent slope at $x_{B}^{(l)}$ close to 1 is very large. Correspondingly, the azeotropic composition is located at a small $x_{B}^{(l)}=0.20$ and the common pressure $h=4.02 \mathrm{kPa}$ is only slightly lower than $p_{A}^{*}$. Furthermore, Fig. 9 shows the phase diagram for the binary mixture of water-tert-butanol with positive deviations, which has an order of $p_{A}^{*}<p_{B}^{*}<K_{A}<K_{B}$, showing overlapping $h_{A}$ and $h_{B}$ ranges. Unlike the binary mixture of acetone-carbon disulfide shown in Fig. 6 with comparable $K_{A}$ and $K_{B}$, here the $K_{B}$ is about $8 \times$ larger than the $K_{A}$ because of a significant positive deviation of tert-butanol's partial pressure from Raoult's law. Nevertheless, the analyses based on eqns 1520 still work very well to locate the azeotrope point at $x_{B}=0.61$ and $h=49.42 \mathrm{kPa}$.

By checking the non-overlapping and overlapping $h$ ranges in Figs. 3-9, one can see that they display three different types of overlapping patterns due to the alignments of the four key quantities $p_{A}^{*}, K_{A}, p_{B}^{*}$, and $K_{B}$. These different alignments are similar to the three kinds of known band alignments in semiconductor heterojunctions, which result from the relative positions of four band edges including the highest edge of the valence band (i.e., valence band maximum) and the lowest edge of the conduction band (i.e., conduction band minimum) for two different semiconductors. The three kinds of band alignments are called straddling (type I), staggered (type II), and broken (type III) ${ }^{31}$ We note that the four key quantities in binary solutions are not related to the four band edges of semiconductor heterojunctions. However, we can borrow the terminologies from the band 
alignments, to help differentiate the different overlapping patterns for $h_{A}$ and $h_{B}$ ranges. Therefore, the non-azeotropic mixture in Figs. 3 and 4 show a broken overlap or no overlap (type III), whereas the azeotropic mixtures in Figs. 5, 8, and 9 show staggered overlap (type II), and the azeotropic mixture in Fig. 6 shows straddling overlap (type I). Only in the type I and type II overlaps a binary mixture can lead to an azeotrope while the type III overlap (or no overlap) will give rise to only non-azeotrope.

3.4. Vapor composition curve for non-azeotropic and azeotropic mixtures. To complete a phase diagram, we need to include the vapor composition curve. When a VLE is established, we have:

$$
\begin{aligned}
& p_{A}=p_{t o t} x_{A}^{(g)}=h_{A} x_{A}^{(l)} \\
& p_{B}=p_{t o t} x_{B}^{(g)}=h_{B} x_{B}^{(l)}
\end{aligned}
$$

Division of the two equations gives:

$$
\frac{x_{A}^{(g)}}{x_{B}^{(g)}}=\frac{h_{A}}{h_{B}} \cdot \frac{x_{A}^{(l)}}{x_{B}^{(l)}}
$$

It appears that eqn 23 can be used to calculate the vapor compositions from the liquid compositions. However, since the values of $h_{A}$ and $h_{B}$ are derived from the vapor compositions, these calculations would form a loop and can only serve the purpose to check whether $h_{A}$ and $h_{B}$ are calculated correctly. Therefore, herein we will just use eqn 23 to show qualitatively where the vapor composition curve should be, simply by using the ratio of $h_{A} / h_{B}$.

Let us consider first non-azeotropic mixtures where the $h_{A}$ and $h_{B}$ ranges do not overlap. If $p_{B}^{*}>p_{A}^{*}$, then it must be true that $h_{B}>h_{A}$. On the basis of eqn 23 , we have the following result regardless of negative or positive deviations from Raoult's law: 


$$
\begin{aligned}
& \frac{x_{A}^{(g)}}{x_{B}^{(g)}}<\frac{x_{A}^{(l)}}{x_{B}^{(l)}} \\
& \frac{1-x_{B}^{(g)}}{x_{B}^{(g)}}<\frac{1-x_{B}^{(l)}}{x_{B}^{(l)}} \\
& x_{B}^{(l)}\left(1-x_{B}^{(g)}\right)<x_{B}^{(g)}\left(1-x_{B}^{(l)}\right) \\
& x_{B}^{(l)}-x_{B}^{(g)} x_{B}^{(l)}<x_{B}^{(g)}-x_{B}^{(g)} x_{B}^{(l)} \\
& x_{B}^{(g)}>x_{B}^{(l)}
\end{aligned}
$$

This means that the vapor composition curve will have more $\mathrm{B}$ component than the liquid counterpart under the same total pressure. Therefore, the vapor composition curve will be on the lower right side of the liquid composition curve (Fig. 1a,c). This is reasonable as component B should be more volatile than component $\mathrm{A}$ due to $p_{B}^{*}>p_{A}^{*}$. Note that the assignment of $\mathrm{A}$ and $\mathrm{B}$ is arbitrary, and therefore the conclusion is also true if $p_{A}^{*}>p_{B}^{*}$. Since the two curves are equal at pure A and pure B, the two curves close together at $x_{A}^{(l)}=0$ and 1 to form a "single-eye-shaped" region, as can be seen from Fig. 1a,c.

For azeotropic mixtures, the $h_{A}$ and $h_{B}$ ranges overlap. At the azeotropic point, according to eqn 19, eqn 23 becomes:

$$
\frac{x_{A}^{(g)}}{x_{B}^{(g)}}=\frac{x_{A}^{(l)}}{x_{B}^{(l)}}
$$

This can be also obtained by using Dalton's law in the following way:

$$
\frac{x_{A}^{(g)}}{x_{B}^{(g)}}=\frac{\frac{n_{A}^{(g)}}{n_{A}^{(g)}+n_{A B}^{(g)}}}{\frac{n_{B}^{(g)}}{n_{A}^{(g)}+n_{A B}^{(g)}}}=\frac{n_{A}^{(g)}}{n_{B}^{(g)}}=\frac{p_{A}}{p_{B}}=\frac{h_{A} x_{A}^{(l)}}{h_{B} x_{B}^{(l)}}=\frac{h_{A}}{h_{B}} \cdot \frac{x_{A}^{(l)}}{x_{B}^{(l)}}=1 \cdot \frac{x_{A}^{(l)}}{x_{B}^{(l)}}=\frac{x_{A}^{(l)}}{x_{B}^{(l)}}
$$

Further simplification of eqn 25 gives:

$$
\frac{x_{A}^{(g)}}{1-x_{A}^{(g)}}=\frac{x_{A}^{(l)}}{1-x_{A}^{(l)}}
$$




$$
\begin{aligned}
& x_{A}^{(g)}\left(1-x_{A}^{(l)}\right)=x_{A}^{(l)}\left(1-x_{A}^{(g)}\right) \\
& x_{A}^{(g)}-x_{A}^{(g)} x_{A}^{(l)}=x_{A}^{(l)}-x_{A}^{(g)} x_{A}^{(l)} \\
& x_{A}^{(g)}=x_{A}^{(l)}
\end{aligned}
$$

and

$$
\begin{aligned}
& 1-x_{B}^{(g)}=1-x_{B}^{(l)} \\
& x_{B}^{(g)}=x_{B}^{(l)}
\end{aligned}
$$

Equations 25-28 indicate that at the azeotropic point, the composition is the same between the vapor and liquid phases.

Next, we consider the full vapor-phase curve on the left and right of the azeotropic point. As have been shown in Fig. 7, for a binary system with negative deviations, when the binary composition is on the left of the azeotropic point, $h_{A}>h_{B}$ or $h_{A} / h_{B}>1$, and when the binary composition is on the right of the azeotropic point, $h_{A}<h_{B}$ or $h_{A} / h_{B}<1$, and of course at the azeotropic composition, $h_{A}=h_{B}$ or $h_{A} / h_{B}=1$, as indicated by eqn 19 . Therefore, by substituting the ratio of $h_{A} / h_{B}$ to eqn 23 , we have the following result on the left of the azeotropic point for a binary system with negative deviation:

$$
\begin{aligned}
& \frac{x_{A}^{(g)}}{x_{B}^{(g)}}>\frac{x_{A}^{(l)}}{x_{B}^{(l)}} \\
& \frac{x_{A}^{(g)}}{1-x_{A}^{(g)}}>\frac{x_{A}^{(l)}}{1-x_{A}^{(l)}} \\
& x_{A}^{(g)}\left(1-x_{A}^{(l)}\right)>x_{A}^{(l)}\left(1-x_{A}^{(g)}\right) \\
& x_{A}^{(g)}-x_{A}^{(g)} x_{A}^{(l)}>x_{A}^{(l)}-x_{A}^{(g)} x_{A}^{(l)} \\
& x_{A}^{(g)}>x_{A}^{(l)}
\end{aligned}
$$

and the following result on the right of the azeotropic point: 


$$
\begin{aligned}
& \frac{x_{A}^{(g)}}{x_{B}^{(g)}}<\frac{x_{A}^{(l)}}{x_{B}^{(l)}} \\
& \frac{1-x_{B}^{(g)}}{x_{B}^{(g)}}<\frac{1-x_{B}^{(l)}}{x_{B}^{(l)}} \\
& x_{B}^{(l)}\left(1-x_{B}^{(g)}\right)<x_{B}^{(g)}\left(1-x_{B}^{(l)}\right) \\
& x_{B}^{(l)}-x_{B}^{(g)} x_{B}^{(l)}<x_{B}^{(g)}-x_{B}^{(g)} x_{B}^{(l)} \\
& x_{B}^{(g)}>x_{B}^{(l)}
\end{aligned}
$$

Therefore, on the left of the azeotropic composition, the vapor phase has larger $x_{A}^{(g)}$ than the liquid phase $x_{A}^{(l)}$ at equilibrium because component $\mathrm{A}$ is more volatile than the azeotrope. On the right of the azeotropic composition, the vapor phase has larger $x_{B}^{(g)}$ than the liquid phase $x_{B}^{(l)}$ at equilibrium because component $\mathrm{B}$ is more volatile than the azeotrope. Here the azeotrope is considered as a single substance instead of a mixture, because its composition remains unchanged during evaporation as indicated by eqns $25-28$. These results dictate that the vapor curve should be lower than the liquid curve and coincides with the liquid-phase curve at the azeotropic point. Finally, it can be readily shown that for a positive azeotrope, the trend is exactly the opposite on the left and right of the azeotrope point, as a result of its opposite ratios of $h_{A} / h_{B}$ except for the point of azeotrope. This can be understood by the fact that the positive azeotrope is more volatile than both component $\mathrm{A}$ and $\mathrm{B}$, contrary to the scenario of the negative azeotrope. These results dictate that the vapor curve should be also lower than the liquid curve and coincides with the liquid-phase curve at the azeotropic point.

Figure 10a,b shows the total vapor pressure as a function of vapor and liquid compositions for two binary mixtures of acetone-chloroform and acetone-carbon disulfide at $35.17{ }^{\circ} \mathrm{C}$ forming negative and positive azeotropes. As mentioned above, the vapor composition curves are below 

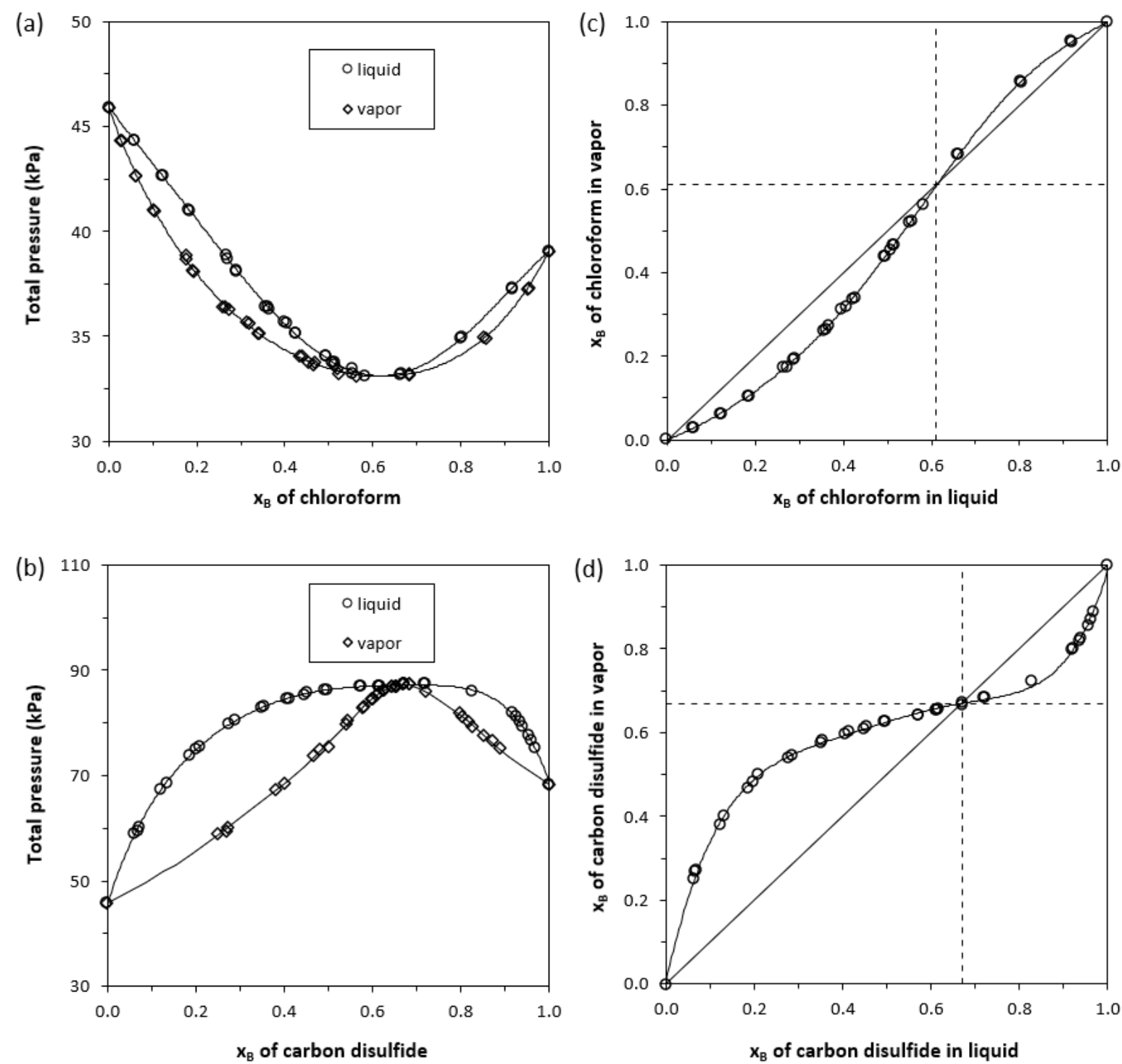

Figure 10. Vapor composition versus liquid composition. $(a, b)$ Total pressure as a function of mole fractions in liquid and vapor. (c,d) Mole fractions in vapor as a function of mole fractions in liquid, for acetone-chloroform $\left\{x_{A} \mathrm{C}_{3} \mathrm{H}_{6} \mathrm{O}+x_{B} \mathrm{CHCl}_{3}\right\}$ and acetone-carbon disulfide $\left\{x_{A} \mathrm{C}_{3} \mathrm{H}_{6} \mathrm{O}\right.$ $\left.+x_{B} \mathrm{CS}_{2}\right\}$ at $308.32 \mathrm{~K}$ forming negative and positive azeotropes (data taken from ref. ${ }^{12,32}$ ). Diagonal line in $(\mathrm{c}, \mathrm{d})$ indicates the situation when vapor and liquid compositions are identical.

the liquid composition curves in both cases, and they have equal mole fractions only for pure A, pure B, and the azeotropic points, thereby forming "double-eye-shaped" regions. It can be further 
seen that in the case of negative deviation, eqns 29 and 30 are satisfied in the two regions on the left and right of the azeotropic point, respectively, while in the case of positive deviation, eqns 29 and 30 are satisfied in the two regions on the right and left of the azeotropic point, respectively. Figure 10c,d further shows the direct comparison between vapor composition and liquid composition. The diagonal line divides the figure into two halves, where the upper left half has $x_{B}^{(g)}>x_{B}^{(l)}$ and the lower right half has $x_{B}^{(g)}<x_{B}^{(l)}$, which is equivalent to $x_{A}^{(g)}>x_{A}^{(l)}$ in eqn 29. Only on the diagonal line, the vapor and liquid compositions are identical, which is the case for pure $\mathrm{A}$, pure $\mathrm{B}$, and the azeotropic points. It can be seen that the azeotropic points divide the figures into two blocks, where eqns 29 and 30 again are satisfied in the lower left block and the upper right block of Fig. 10c, respectively, and in the upper right block and the lower left block of Fig. 10d, respectively.

With both the liquid and vapor compositions shown in Fig. 10a,b, we further justify why an azeotrope should be considered as a single substance instead of a mixture, despite the fact it is made up of two components. On the isothermal phase diagram for each binary system, there is only one azeotropic point with a fixed combination of pressure and composition. Similar to the cases of pure components A and B, the degree of freedom or variance for an azeotrope is $f=3-\Phi$ $=3-2=1$ according to the Gibbs phase rule at the vapor pressure of $h_{A}=h_{B}=h$ due to coexisting two phases of vapor and liquid. Alternatively, the conditional degree of freedom with temperature fixed is $f^{*}=3-1-\Phi=2-\Phi=2-2=0$. This means that for an azeotrope, once temperature is fixed, then the pressure and composition are not variables. If the azeotrope is forced to be two components, then its Gibbs phase rule should be $\mathrm{f}=4-\Phi=4-2=2$ or $\mathrm{f}^{*}=4-1-\Phi$ $=3-\Phi=3-2=1$, which does not agree with the fact that the pressure and mole fraction are fixed for azeotrope under a certain temperature. Therefore, an azeotrope must be considered a 
single substance. In comparison, in one of the two eye-shaped regions, the conditional degree of freedom is $f^{*}=3-2=1$, which means that a pressure is needed to define the system because under this pressure the compositions of vapor and liquid can be found from the phase diagram; under the vapor composition curve or above the liquid composition curve, the conditional degree of freedom is $\mathrm{f}^{*}=3-1=2$, which means both pressure and composition are needed to fully define the system.

\section{Conclusion}

We have put forth a simple and yet practical theoretical model generalized from Raoult's law and Henry's law. The model entertains a hybrid parameter $h_{B}$ that is related to the experimentally accessible activity coefficient. Throughout the discussions the hybrid parameter $h_{B}$ plays a central role. The values of $h_{B}$ are mole fraction dependent and fall in the range of $\left[K_{B}, p_{B}^{*}\right]$ or $\left[p_{B}^{*}, K_{B}\right]$ in the case of negative or positive deviation from Raoult's law, respectively. When the mole fraction approaches 1 and 0 , the hybrid parameter $h_{B}$ asymptotically approaches the limiting values of $p_{B}^{*}$ and $K_{B}$, respectively. Under the same mole fraction limits, the generalized model can be reduced to the Raoult's law and Henry's law, respectively.

We have uncovered an overlapping rule for the ranges of $h_{A}$ and $h_{B}$, which binary mixtures must obey to form azeotropes. There can be three different types of overlapping patterns, i.e., straddling overlap (type I), staggered overlap (type II), and broken overlap or no overlap (type III). For binary mixtures with either type I or type II overlaps, azeotropes are formed when the $h_{A}$ at a mole fraction for component $\mathrm{A}$ is equal to the $h_{B}$ at a corresponding mole fraction for component $\mathrm{B}$, giving a total pressure at common $h$ for the azeotrope composition, regardless of negative or positive deviations. For binary mixtures with type III overlap (or no overlap), non-azeotropes are 
formed. For ideal solutions, the $h_{A}$ and $h_{B}$ ranges are condensed to just two different points with non-overlapping pressures, and therefore ideal solutions do not form azeotropes. For the ranges of $h_{A}$ and $h_{B}$ to overlap, the intermolecular interactions $\Delta E$ should be stronger than $\left|\ln \left(p_{A}^{*} / p_{B}^{*}\right)\right| k_{B} T$. This overlapping rule serves as a simple guideline to rationalize why some solutions form azeotropes while others do not. It is worth pointing out that only the ranges of the hybrid parameter $h$ work for this purpose, while those of activity $\alpha$ and activity coefficient $\gamma$ do not.

We have also shown a semi-quantitative way to rationalize the characteristic mole fraction and pressure for binary azeotropes. In isothermal phase diagrams, one can first draw an auxiliary horizontal line tangent with the concave or convex total pressure curve at the minimum or maximum pressure for negative or positive deviations, respectively. This horizontal line intersects with the two vertical axes at $h_{A}$ on the left and $h_{B}$ on the right, which is equal to the common pressure $h$ for the azeotrope. One can then connect point $h_{A}$ with point $\mathrm{B}$ at zero pressure and connect point $h_{B}$ with point $\mathrm{A}$ at zero pressure. These lines will intersect with the partial pressure curves for the two components at the azeotropic mole fraction. Finally, a vertical line can be drawn at the azeotropic mole fraction to intersect with the horizontal line to find the azeotropic point.

Finally, we have presented a qualitative interpretation for the relative mole fractions of vapor composition curves with respect to the liquid counterparts under the same pressures. For nonazeotropic mixtures where the $h_{A}$ and $h_{B}$ ranges do not overlap, the vapor composition curve will have more B component (if $h_{B}>h_{A}$ ) or more A component (if $h_{A}>h_{B}$ ) than the liquid counterpart under the same total pressure. For azeotropic mixtures with negative deviation, we have $h_{A}>h_{B}$ or $h_{A}<h_{B}$ if the binary composition is on the left or right of the azeotropic point, respectively, and $h_{A}=h_{B}$ at the azeotropic composition. Therefore, on the left or right of the azeotropic composition, the vapor phase has more component A or B than the liquid phase because 
component $\mathrm{A}$ or $\mathrm{B}$ is more volatile than the azeotrope, whereas at the azeotropic point, the composition is the same between the vapor and liquid phases. The opposite scenarios can be obtained for a binary system with positive deviation, except for the point of azeotrope.

To the best of our knowledge, the understandings presented in this work have not been adopted for the analysis of realistic experimental data or documented in any physical chemistry or chemical engineering books. We believe the approaches presented in this work will be useful in the analysis of experimental data from VLE measurements and also possess pedagogical merit in various relevant fields. For instance, in the data analysis presented in the Supplementary Materials for the binary dimethoxymethane-carbon disulfide solution, we noticed that the experimental data are inconsistent between the azeotropic mole fraction $\left(x_{B}=0.48\right)$ and the maximum pressure position on the total pressure curve $\left(x_{B}=0.45\right)$, which can be ascribed to the relatively large uncertainty of $\pm 0.05 \mathrm{kPa}$ in pressure measurement. This illustrates how the understandings of the present work can be used as a framework to analyze and calibrate experimental results. In addition, eqns 12-16 and 19-20 along with the tangent lines and auxiliary lines shown in Figs. 5-9 can be adopted to explain fundamental concepts in the textbook of Atkins' Physical Chemistry, in the open access textbook in the chemistry library of the LibreTexts project, and in the LearnChemE chemical engineering education resources.

Acknowledgment: This work was performed at Oak Ridge National Laboratory's Center for Nanophase Materials Sciences, a U.S. DOE Office of Science User Facility. J.H. and D.S.S. gratefully acknowledge the support from the Laboratory Directed Research and Development Program of ORNL. 
Supplementary Materials Available: An excel file with detailed steps for data analysis using the experimental data of binary dimethoxymethane-carbon disulfide solution $\left\{x_{A} \mathrm{C}_{3} \mathrm{H}_{8} \mathrm{O}_{2}+x_{B} \mathrm{CS}_{2}\right\}$ at $308.32 \mathrm{~K}$. Additional statistical mechanics explanations of activity $\alpha_{B}$, activity coefficient $\gamma_{B}$, and the hybrid parameter $h_{B}$ in terms of intermolecular interactions related parameter $\beta$.

\section{References:}

${ }^{1}$ Schlünder, E.-U. Azeotropes and Pseudo-Azeotropes. Fluid Phase Equilib. 1989, 51, 71-85.

${ }^{2}$ Kiva, V. N.; Hilmen, E. K.; Skogestad, S. Azeotropic Phase Equilibrium Diagrams: A Survey. Chem. Eng. Sci. 2003, 58, 1903-1953.

${ }^{3}$ Brady, J. E. General Chemistry: Principles \& Structure, $5^{\text {th }}$ ed.; John Wiley \& Sons, 1990.

${ }^{4}$ National Institute of Standards and Technology, Standard Reference Material 1828: EthanolWater Solutions. https://www-s.nist.gov/srmors/certificates/archives/1828.pdf.

${ }^{5}$ Izadi, H.; Focke, W. W.; Asaadi, E.; Maharaj, R.; Pretorius, J.; Loots, M. T. A Promising Azeotrope-Like Mosquito Repellent Blend. Sci. Rep. 2017, 7, 10273.

${ }^{6}$ Handbook for Critical Cleaning: Cleaning agents and systems, $2^{\text {nd }}$ ed.; Kanegsberg, B, Kanegsberg, E., Eds.; CRC Books, Boca Raton, USA, 2011.

${ }^{7}$ Valentini, F.; Vaccaro, L. Azeotropes as Powerful Tool for Waste Minimization in Industry and Chemical Processes. Molecules 2020, 25, 5264.

${ }^{8}$ Wistreich, H. E.; Blake, J. A. Azeotropic Freeze-Drying. Science 1962, 138, 138.

${ }^{9}$ Qi, J.; Zeng, F.; Jia, H.; Fang, J.; Li, H.; Wen, X.; Fang, Z. Research Progress on the Formation Mechanism of Azeotrope and its Separation Process in Microwave Field. J. Chem. Technol. Biotechnol. 2021, Early View. 
10 Becker, F.; Kiefer, M.; Rhensius, P.; Schaefer, H.-D. Interpretation von Dampfdruckdiagrammen binärer flüssiger Mischungen mit Hilfe von Gleichgewichtsmodellen. Systeme Diäthyläther-Chloroform, Diäthylaether-Tetrachlorkohlenstoff, TetrahydrofuranTetrachlorkohlenstoff und Hexafluorbenzol-Benzol. Z. Physik. Chem. 1974, 92, 169-192.

${ }^{11}$ Puri, P. S.; Ruether, J. A. Vapor-Liquid Equilibria of Acetone-Cyclohexane and Acetonelsopropanol Systems at $25^{\circ}$ C. J. Chem. Eng. Data. 1974, 19, 87-89.

${ }^{12}$ von Zawidzki, J. Über die Dampfdrücke binärer Flüssigkeitsgemische, Z. Physik. Chem. 1900, $35,129-203$.

${ }^{13}$ Hadler, A. B.; Ott, L. S.; Bruno, T. J. Study of Azeotropic Mixtures with the Advanced Distillation Curve Approach. Fluid Phase Equilib. 2009, 281, 49-59.

${ }^{14}$ Shephard, J. J.; Callear, S. K.; Imberti, S.; Evans, J. S. O.; Salzmann, C. G. Microstructures of Negative and Positive Azeotropes. Phys. Chem. Chem. Phys. 2016, 18, 19227.

${ }^{15}$ Rushbrooke, G. S. Introduction to Statistical Mechanics; Oxford University Press, 1949.

${ }^{16}$ Hadj-Kali, M. K.; Gerbaud, V.; Joulia, X. Azeotrope Prediction by Monte Carlo Molecular Simulation. Chem. Eng. Commun. 2012, 199, 673-688.

${ }^{17}$ Li, D.; Gao, Z.; Vasudevan, N. K.; Li, H.; Gao, X.; Li, X.; Xi, L. Molecular Mechanism for Azeotrope Formation in Ethanol/Benzene Binary Mixtures through Gibbs Ensemble Monte Carlo Simulation. J. Phys. Chem. B 2020, 124, 3371-3386.

${ }^{18}$ Atkins, P.; de Paula, J. Atkins' Physical Chemistry, $8^{\text {th }}$ ed.; W. H. Freeman and Company, 2006.

${ }^{19}$ Open access textbook in the chemistry library of the LibreTexts project. See https://chem. libretexts.org/Bookshelves/Physical_and_Theoretical_Chemistry_Textbook_Maps/DeVoes_Ther modynamics and Chemistry/13\%3A The Phase_Rule and Phase_Diagrams/132 Phase Diagr ams\%3A_Binary_Systems. 
${ }^{20}$ Wolfram Demonstration Project, Vapor Pressures of Binary Solutions. See https://demonstrations.wolfram.com/VaporPressuresOfBinarySolutions/ and https://learncheme.com/simulations/ thermodynamics/thermo-2/vapor-pressure-of-binary-solutions/.

${ }^{21}$ Wichterle, I.; Linek, J.; Wagner, Z.; Fontaine, J.-C.; Sosnkowska-Kehiaian, K.; Kehiaian, H. V. Lanbolt-Börnstein Numerical Data and Functional Relationships in Science and Technology, Group IV: Physical Chemistry, Vol. 13: Vapor-Liquid Equilibrium in Mixtures and Solutions, Subvolume A - Binary Liquid Systems of Nonelectrolytes Part 2; Kehiaian, H. V., Ed.; Springer, 2008; LB5412.

${ }^{22}$ Ozog, J. Z.; Morrison, J. A. Activity Coefficients of Acetone-Chloroform Solutions. J. Chem. Ed. 1983, 60, 72-74.

${ }^{23}$ Ott, J. B.; Boerio-Goates, J. Chapter 6: Fugacity, Activity, and Standard States. In Chemical Thermodynamics: Principles and Applications; Elsevier, San Diego, 2000.

${ }^{24}$ Yin, X.-B. Relationship and Difference among Raoult's Law, Henry's Law, and Azeotropic Solution. Univ. Chem. 2018, 33, 61-65.

${ }^{25}$ Wichterle, I.; Linek, J.; Wagner, Z.; Fontaine, J.-C.; Sosnkowska-Kehiaian, K.; Kehiaian, H. V. Lanbolt-Börnstein Numerical Data and Functional Relationships in Science and Technology, Group IV: Physical Chemistry, Vol. 13: Vapor-Liquid Equilibrium in Mixtures and Solutions, Subvolume A - Binary Liquid Systems of Nonelectrolytes Part 2; Kehiaian, H. V., Ed.; Springer, 2008; LB5600.

${ }^{26}$ The derivation was shown as $\frac{n_{A}^{(l)}}{n_{B}^{(l)}}=\frac{n_{A}^{(g)}}{n_{B}^{(g)}}=\frac{p_{A}}{p_{B}}=\frac{K_{A}^{\prime} \times x_{A}^{(l)}}{K_{B}^{\prime} \times x_{B}^{(l)}}=\frac{K_{A}^{\prime} \times\left[n_{A}^{(l)} /\left(n_{A}^{(l)}+n_{B}^{(l)}\right)\right]}{K_{B}^{\prime} \times\left[n_{B}^{(l)} /\left(n_{A}^{(l)}+n_{B}^{(l)}\right)\right]}=\frac{K_{A}^{\prime} \times n_{A}^{(l)}}{K_{B}^{\prime} \times n_{B}^{(l)}}=\frac{K_{A}^{\prime}}{K_{B}^{\prime}} \times$ $\frac{n_{A}^{(l)}}{n_{B}^{(l)}}$. To be more exact, the derivation can be $\frac{x_{A}^{(l)}}{x_{B}^{(l)}}=\frac{x_{A}^{(g)}}{x_{B}^{(g)}}=\frac{n_{A}^{(g)} /\left(n_{A}^{(g)}+n_{B}^{(g)}\right)}{n_{B}^{(g)} /\left(n_{A}^{(g)}+n_{B}^{(g)}\right)}=\frac{n_{A}^{(g)}}{n_{B}^{(g)}}=\frac{p_{A}}{p_{B}}=\frac{K_{A}^{\prime} \times x_{A}^{(l)}}{K_{B}^{\prime} \times x_{B}^{(l)}}=$ $\frac{K_{A}^{\prime}}{K_{B}^{\prime}} \times \frac{x_{A}^{(l)}}{x_{B}^{(l)}}$. However, $\frac{n_{A}^{(l)}}{n_{B}^{(l)}}=\frac{n_{A}^{(g)}}{n_{B}^{(g)}}$ or $\frac{x_{A}^{(l)}}{x_{B}^{(l)}}=\frac{x_{A}^{(g)}}{x_{B}^{(g)}}$ is a conclusion rather than a starting condition for 
azeotropes. In addition, there are three points on the phase diagram that satisfy this condition, including the pure components $\mathrm{A}$ and $\mathrm{B}$ in addition to the azeotropic composition. The conclusion of $K_{A}^{\prime}=K_{B}^{\prime}$ holds true only for the azeotropic composition where $\frac{x_{A}^{(l)}}{x_{B}^{(l)}}$ is not $\infty$ (pure A) or 0 (pure B).

${ }^{27}$ Mueller, C. R.; Kearns, E. R. Thermodynamic Studies of the System: Acetone + Chloroform. J. Phys. Chem. 1958, 62, 1441-1445.

${ }^{28}$ Handa, Y. P.; Fenby, D. V.; Jones, D. E. Vapour Pressures of Triethyiamine + Chloroform and of Triethylamine + Dichloromethane. J. Chem. Thermodyn. 1975, 7, 337-343.

${ }^{29}$ Koga, Y. Chapter II: Solution Thermodynamics - Use of the Second and Third Derivatives of G. In Solution Thermodynamics and Its Application to Aqueous Solution-A differential Approach; Elsevier, 2007.

${ }^{30}$ Koga, Y.; Siu, W. W. Y.; Wong, T. Y. H. Excess Partial Molar Free Energies and Entropies in Aqueous tert-Butyl Alcohol Solutions at $25^{\circ}$ C. J. Phys. Chem. 1990, 94, 7700-7706.

${ }^{31}$ Selinsky, R. S.; Ding, Q.; Faber, M. S.; Wright, J. C.; Jin, S. Quantum Dot Nanoscale Hetreostructures for Solar Energy Conversion. Chem. Soc. Rev. 2013, 42, 2963-2985.

${ }^{32}$ Wichterle, I.; Linek, J.; Wagner, Z.; Fontaine, J.-C.; Sosnkowska-Kehiaian, K.; Kehiaian, H. V. Lanbolt-Börnstein Numerical Data and Functional Relationships in Science and Technology, Group IV: Physical Chemistry, Vol. 13: Vapor-Liquid Equilibrium in Mixtures and Solutions, Subvolume A - Binary Liquid Systems of Nonelectrolytes Part 2; Kehiaian, H. V., Ed.; Springer, 2008; LB5441 and LB5411. 